\title{
WITT'S EXTENSION THEOREM FOR QUADRATIC SPACES OVER SEMIPERFECT RINGS
}

\author{
URIYA A. FIRST
}

\begin{abstract}
We prove that every isometry of between (not-necessarily orthogonal) summands of a unimodular quadratic space over a semiperfect ring can be extended an isometry of the whole quadratic space. The same result was proved by Reiter for the broader class of semilocal rings, but with certain restrictions on the base modules, which cannot be removed in general.

Our result implies that unimodular quadratic spaces over semiperfect rings cancel from orthogonal sums. This improves a cancellation result of Quebbemann, Scharlau and Schulte, which applies to quadratic spaces over hermitian categories. Combining this with other known results yields further cancellation theorems. For instance, we prove cancellation of (1) systems of sesquilinear forms over henselian local rings, and (2) non-unimodular hermitian forms over (arbitrary) valuation rings.

Finally, we determine the group generated by the reflections of a unimodular quadratic space over a semiperfect ring.
\end{abstract}

\section{INTRODUCTION}

Let $F$ be a field of characteristic not 2 and let $(V, q)$ be a nondegenerate quadratic space over $F$. The following theorem, known as Witt's Theorem or Witt's Extension Theorem, is fundamental in the theory of quadratic forms.

Theorem 1.1 (Witt). Let $V_{1}, V_{2}$ be subspaces of $V$ and let $\psi:\left.\left.q\right|_{V_{1}} \rightarrow q\right|_{V_{2}}$ be an isometry. Then $\psi$ extends to an isometry $\psi^{\prime}$ of $q$. Furthermore, $\psi^{\prime}$ is a product of reflections.

Among the theorem's consequences are cancellation of nondegenerate quadratic spaces and the fact that $O(V, q)$, the isometry group of $(V, q)$, acts transitively on maximal totally isotropic subspaces of $V$.

The works of Bak [2, Wall [26] and others have led to defining a notion of quadratic forms over arbitrary (non-commutative) rings, and also to an appropriate definition of reflections (see [12, [14, 20]). In this context, Witt's Extension Theorem was generalized by Reiter 20 to semilocal rings, but with certain restrictions on the quadratic spaces (see also 12 and 14 for earlier results). Cancellation of unimodular quadratic spaces was likewise generalized to various families of semilocal rings $A$ including the cases where $A$ is commutative ([22, [12] or [11, §3.4.3]), local ([11, Rm. 3.4.2], for instance), or $A=\varliminf_{\varlimsup}\left\{A / \operatorname{Jac}(A)^{n}\right\}_{n \in \mathbb{N}}([19, \S 3.4]$; this case includes all one-sided artinian rings). However, despite the previous evidence,

Date: September 7, 2018.

Key words and phrases. quadratic form, Witt's Theorem, reflection, isometry group, semiperfect ring, semilocal ring, Dickson's invariant, hermitian category, sesquilinear form.

The author was supported by an ERC grant \#226135 and an SNFS grant \#IZK0Z2_151061. 
Keller [1] has demonstrated that cancellation fails over arbitrary semilocal rings, implying that the restrictions in Reiter's Theorem cannot be removed in general. (Most of the results mentioned here can also be found in [15, Ch. VI].)

In this paper, we restrict our attention to a family of semilocal rings called semiperfect rings, and study to what extent Witt's Extension Theorem holds in this setting. Recall that a ring $A$ is called semiperfect if it is semilocal and its Jacobson radical, $\operatorname{Jac}(A)$, is idempotent lifting. For example, local rings and semilocal rings satisfying $A=\lim _{\lfloor}\left\{A / \operatorname{Jac}(A)^{n}\right\}_{n \in \mathbb{N}}$ are semiperfect. See $\S 2.5$ below for further examples and details.

Let $(P,[\beta])$ be a unimodular quadratic space over a semiperfect ring (the definition is recalled below) and let $Q, S$ be summands of $P$. Our main results are:

(1) Every isometry $\left(Q,\left[\left.\beta\right|_{Q}\right]\right) \rightarrow\left(S,\left[\left.\beta\right|_{S}\right]\right)$ extends to an isometry of $(P,[\beta])$ (Corollary 4.9, Theorem 4.8).

(2) Under mild assumptions, every isometry of $(P,[\beta])$ is a product of quasireflections (Corollary 4.11, Theorem 4.5).

(3) We determine the subgroup of $O(P,[\beta])$ generated by reflections (Theorem 5.8). Apart from an obvious exception in which there are no reflections, this subgroup is always of finite index in $O(P,[\beta])$.

The proofs are based on Reiter's ideas with certain improvements. In particular, we generalize Reiter's e-reflections (see [20]). We also stress that (1)-(3) hold without assuming 2 is invertible.

Our results imply that unimodular quadratic spaces over semiperfect rings cancel from orthogonal sums. (Note that the base ring in Keller's counterexample [11, §2] is semilocal but not semiperfect.) This in turn leads to other cancellation theorems as follows: In [5, 7] and [6, it was shown that systems of (not-necessarily unimodular) sesquilinear forms can be treated as (single) unimodular hermitian forms over a different base ring. Thus, cancellation holds when this base ring is semiperfect. Using this, we show that cancellation holds for

(a) arbitrary (i.e. not-necessarily unimodular) hermitian forms over involutary valuation rings (Corollary 4.18),

(b) systems of sesquilinear forms over involutary henselian valuation rings (Corollary 4.17).

We also strengthen a cancellation theorem of Quebbeman, Scharlau and Schulte 19 , $\S 3.4]$ which applies to quadratic spaces over hermitian categories (Corollary 4.14). Specifically, the cancellation of [19, §3.4] assumes that the underlying hermitian category satisfies: (i) all idempotents split, (ii) every object is the direct sum of objects with local endomorphism ring, and (iii) if $A$ is the endomorphism ring of an object, then $A=\lim _{\longleftarrow}\left\{A / \operatorname{Jac}(A)^{n}\right\}_{n \in \mathbb{N}}$. We show that cancellation holds even without assuming condition (iii).

The paper is organized as follows: In section 2, we recall the definitions of quadratic forms over rings and several results to be used throughout. Section 3 introduces quasi-reflections and reflections. In section 4, we prove our version of Witt's Extension Theorem and discuss its applications. Finally, in section 5 , we describe the group spanned by the reflections of a unimodular quadratic space (over a semiperfect ring). 


\section{Preliminaries}

This section collects several preliminary topics that will be used throughout the paper: We recall quadratic forms over unitary rings, several facts concerning them, a notion of orthogonality for unitary rings, and several facts about semiperfect rings.

$\S 2.1$. Quadratic Forms. We start with recalling quadratic forms. The definitions go back to Bak [2] and Wall [26. See [3], [23, Ch. 7], or [15] for an extensive discussion.

Let $A$ be a ring. An anti-structure on $A$ consists of a pair $(\sigma, u)$ such that $\sigma: A \rightarrow A$ is an anti-automorphism (written exponentially) and $u \in A^{\times}$satisfies $u^{\sigma} u=1$ and $a^{\sigma \sigma}=u a u^{-1}$ for all $a \in A$.

Denote by $\mathscr{P}(A)$ the category of finitely generated projective right $A$-modules. As usual, a sesquilinear space is a pair $(P, \beta)$ such that $P \in \mathscr{P}(A)$ and $\beta: P \times P \rightarrow$ $A$ is a biadditive map satisfying $\beta(x a, y b)=a^{\sigma} \beta(x, y) b$ for all $x, y \in P, a, b \in A$. In this case, we call $\beta$ a sesquilinear form. The form $\beta$ is called $u$-hermitian if it also satisfies $\beta(x, y)=\beta(y, x)^{\sigma} u$.

We say that $(P, \beta)$ is unimodular if the map $L_{\beta}: P \rightarrow P^{*}:=\operatorname{Hom}_{A}(P, A)$ given by sending $x \in P$ to $[y \mapsto \beta(x, y)] \in P^{*}$ is an isomorphism. Note that $P^{*}$ can be made into a right $A$-module by setting $(f a) x=a^{\sigma}(f x)$ for all $f \in P^{*}, a \in A, x \in P$. This makes $L_{\beta}$ is a homomorphism of $A$-modules. There is a natural isomorphism $\omega_{P}: P \rightarrow P^{* *}$ give by $\left(\omega_{P} f\right) x=(f x)^{\sigma} u$ for all $f \in P^{*}, x \in P$.

Next, set $\Lambda^{\min }=\left\{a-a^{\sigma} u \mid a \in A\right\}$ and $\Lambda^{\max }=\left\{a \in A: a^{\sigma} u=-a\right\}$. A form parameter (for $(A, \sigma, u)$ ) consists of an additive group $\Lambda$ such that

$$
\Lambda^{\min } \subseteq \Lambda \subseteq \Lambda^{\max } \quad \text { and } \quad a^{\sigma} \Lambda a \subseteq \Lambda \quad \forall a \in A
$$

In this case, the quartet $(A, \sigma, u, \Lambda)$ is called a unitary ring.

For $P \in \mathscr{P}(A)$, let $\mathcal{S}_{P}$ denote the abelian group of sesquilinear forms on $P$, and let $\Lambda_{P}$ denote the subgroup consisting of sesquilinear forms $\gamma \in \mathcal{S}_{P}$ satisfying $\gamma(x, y)=-\gamma(y, x)^{\sigma} u$ and $\gamma(x, x) \in \Lambda$ for all $x, y \in P$. The image of $\beta \in \mathcal{S}_{P}$ in $\mathcal{S}_{P} / \Lambda_{P}$ is denoted $[\beta]$.

A quadratic space (over $(A, \sigma, u, \Lambda))$ is a pair $(P,[\beta])$ with $P \in \mathscr{P}(A)$ and $[\beta] \in$ $\mathcal{S}_{P} / \Lambda_{P}$. Associated with $[\beta]$ are the $u$-hermitian form

$$
h_{\beta}(x, y)=\beta(x, y)+\beta(y, x)^{\sigma} u
$$

and the quadratic map $\hat{\beta}: P \rightarrow A / \Lambda$ given by

$$
\hat{\beta}(x)=\beta(x, x)+\Lambda .
$$

Both $h_{\beta}$ and $\hat{\beta}$ are determined by the class $[\beta]$, and conversely, $[\beta]$ is determined by $h_{\beta}$ and $\hat{\beta}$. We also have

$$
\hat{\beta}(x+y)=\hat{\beta}(x)+\hat{\beta}(y)+h_{\beta}(x, y) \quad \forall x, y \in P .
$$

We say that $(P,[\beta])$ is unimodular if $h_{\beta}$ is a unimodular $u$-hermitian form, i.e. if $L_{[\beta]}:=L_{h_{\beta}}$ is an isomorphism ( $\beta$ itself may be non-unimodular).

Isometries between quadratic (resp. sesquilinear, $u$-hermitian) spaces are defined in the standard way (cf. [15, $\S \mathrm{I} .2 .2, \S \mathrm{I} .5 .2]$ ). We let $O(P,[\beta])$ denote the group of isometries of $(P,[\beta])$. The category of unimodular quadratic spaces over $(A, \sigma, u, \Lambda)$ (with isometries as morphisms) is denoted by $\mathrm{UQ}^{u, \Lambda}(A, \sigma)$. 
Remark 2.1. When $2 \in A^{\times}$, we have $\Lambda^{\min }=\Lambda^{\max }$, so there is only one form parameter $\Lambda$. Furthermore, in this case, $\mathrm{UQ}^{u, \Lambda}(A, \sigma)$ is isomorphic to the the category of unimodular $u$-hermitian forms over $(A, \sigma, u)$. Indeed, $[\beta]$ can be recovered from $h_{\beta}$ via $[\beta]=\left[\frac{1}{2} h_{\beta}\right]$.

Remark 2.2. The "classical" notion of a quadratic space over a commutative ring $A$ (see [22, for instance) occurs in the special case $\sigma=\operatorname{id}_{A}, u=1$ and $\Lambda=0$. Then, the quadratic map $\hat{\beta}$ determines $h_{\beta}$ by (2.1).

Remark 2.3. The triple $\left(\mathscr{P}(A), *,\left\{\omega_{P}\right\}_{P \in \mathscr{P}(A)}\right)$ is a hermitian category and the pair $\left(1, \underline{\Lambda}:=\left\{\Lambda_{P}\right\}_{P \in \mathscr{P}(A)}\right)$ is a form parameter (see [19] or [15, $\left.\S I I .2\right]$ for the definitions). There is a one-to-one correspondence between quadratic spaces over $(A, \sigma, u, \Lambda)$ and quadratic spaces over $(\mathscr{P}(A), 1, \underline{\Lambda})$ given by $(P,[\beta]) \mapsto\left(P,\left[L_{\beta}\right]\right)$.

$\S 2.2$. Conjugation and Transfer. We now introduce two well-known procedures that we refer to as conjugation and e-transfer. They allow one to alter a unitary ring $(A, \sigma, u, \Lambda)$ while maintaining data about isometries between quadratic forms (isometry groups in particular). We shall use these manipulations several times in the sequel.

Proposition 2.4 ("Conjugation"). Let $v \in A^{\times}$. Define $\left(\sigma^{\prime}, u^{\prime}, \Lambda^{\prime}\right)$ by

$$
a^{\sigma^{\prime}}=v a^{\sigma} v^{-1}, \quad u^{\prime}=v\left(v^{\sigma}\right)^{-1} u, \quad \Lambda^{\prime}=v \Lambda .
$$

We call $\left(\sigma^{\prime}, u^{\prime}, \Lambda^{\prime}\right)$ the conjugation of $(\sigma, u, \Lambda)$ by $v$. Then $\left(A, \sigma^{\prime}, u^{\prime}, \Lambda^{\prime}\right)$ is a unitary ring and $\mathrm{UQ}^{u, \Lambda}(A, \sigma) \cong \mathrm{UQ}^{u^{\prime}, \Lambda^{\prime}}\left(A, \sigma^{\prime}\right)$.

Proof. This proposition is essentially [20, Lm. 1.6]; everything follows by straightforward computation. The categorical equivalence is constructed as follows: For any $P \in \mathscr{P}(A)$ and $\beta \in \mathcal{S}_{P}$, define $v \beta: P \times P \rightarrow A$ by

$$
(v \beta)(x, y)=v \cdot \beta(x, y) .
$$

Then $(P, v \beta)$ is s sesquilinear form over $\left(A, \sigma^{\prime}\right)$, and the assignment $(P,[\beta]) \mapsto$ $(P,[v \beta])$ defines the required categorical isomorphism (isometries are mapped to themselves).

Proposition 2.5 ("e-transfer"). Let $e \in A$ be an idempotent satisfying $e^{\sigma}=e$ and Ae $A=A$. For every sesquilinear form $\beta: P \times P \rightarrow A$, denote by $\beta_{e}$ the restriction of $\beta$ to $\mathrm{Pe} \times \mathrm{Pe}$. Then:

(i) $(B, \tau, v, \Gamma):=\left(e A e,\left.\sigma\right|_{e A e}, e u, e \Lambda e\right)$ is a unitary ring.

(ii) $\left(P e,\left[\beta_{e}\right]\right)$ is a quadratic space over $(B, \tau, v, \Gamma)$. It is unimodular if and only if $(P,[\beta])$ is unimodular.

(iii) The map $[\beta] \mapsto\left[\beta_{e}\right]: \mathcal{S}_{P} / \Lambda_{P} \rightarrow \mathcal{S}_{P e} / \Lambda_{P e}$ is an abelian group isomorphism. In particular, $[\beta]=[0] \Longleftrightarrow\left[\beta_{e}\right]=[0]$.

(iv) The assignment $(P,[\beta]) \mapsto\left(P e,\left[\beta_{e}\right]\right)$, called e-transfer, gives rise to an equivalence of categories $\mathrm{UQ}^{u, \Lambda}(A, \sigma) \sim \mathrm{UQ}^{v, \Gamma}(B, \tau)$.

Proof (sketch). Part (i) is straightforward.

For parts (ii), (iii) and (iv), view $\mathscr{P}(A)$ and $\mathscr{P}(B)$ as hermitian categories with a form parameter as in Remark 2.3. Let $F: \mathscr{P}(A) \rightarrow \mathscr{P}(B)$ be the functor given by $F P=P \otimes_{A} A e \cong P e$. By Morita Theory (see [18, $\left.\S 18 \mathrm{D}\right]$ for instance), $F$ is an equivalence of categories. In addition, there is a natural isomorphism $\phi_{P}$ from $F\left(P^{*}\right)=P^{*} e$ to $(F P)^{*}=\operatorname{Hom}_{e A e}(P e, e A e)$ given by $\left.\phi(f) \mapsto f\right|_{P e}$ (check this for 
$P=A_{A}$, the general case follows by additivity). It is routine to check that $(F, \phi)$ is strictly duality preserving functor from $\mathscr{P}(A)$ to $\mathscr{P}(B)$ (see [19, §2]). This means parts (ii) and (iii) hold tautologically, and part (iv) follows from [19, Lm. 2.1] (for instance).

$\S 2.3$. Simple Unitary Rings. A unitary $\operatorname{ring}(A, \sigma, u, \Lambda)$ is called simple if the only ideals of $A$ which are invariant under $\sigma$ are 0 and $A$. It is not hard to show that in this case, $A$ is either simple, or $A \cong B \times B^{\text {op }}$, where $B$ is a simple ring, and $\sigma$ is given by $\left(a, b^{\mathrm{op}}\right)^{\sigma}=\left(t b t^{-1}, a^{\mathrm{op}}\right)$ for some $t \in B^{\times}$.

Assume $(A, \sigma, u, \Lambda)$ is simple and $A$ is artinian. Then the Artin-Wedderburn Theorem implies that $A \cong \mathrm{M}_{n}(D)$ where $D$ is a division ring or a product of a division ring and its opposite. Identifying $A$ with $\mathrm{M}_{n}(D)$, we say that such $(A, \sigma, u, \Lambda)$ is standard or in standard form if:

(1) $\sigma$ is of the form $\left(d_{i j}\right)_{i, j} \mapsto\left(d_{j i}^{\tau}\right)_{i, j}$ for some involution $\tau: D \rightarrow D$. (In particular, $\sigma$ is an involution.)

(2) When $D \cong E \times E^{\text {op }}$ with $E$ a division ring, $\tau$ is the exchange involution $\left(a, b^{\mathrm{op}}\right) \mapsto\left(b, a^{\mathrm{op}}\right)$.

(3) $u=1$ if $\tau \neq \operatorname{id}_{D}$.

In this case, we have $u \in\{ \pm 1\}$ (because when $\tau=\operatorname{id}_{D}$, we have $u^{2}=u^{\sigma} u=1$ ).

It is not true that any simple artinian unitary ring is isomorphic to a unitary ring in standard form. However, this is true after applying a suitable conjugation in the sense of Proposition 2.4, and conjugation does not essentially change the category of quadratic spaces.

Proposition 2.6. After a suitable conjugation (cf. Proposition 2.4), any simple artinian unitary ring $(A, \sigma, u, \Lambda)$ is isomorphic to a unitary ring in standard form.

Proof. Apart from a small difference in condition (3), this proposition is [20, Pr. 2.1]. We have included here a full proof of the sake of completeness.

Let $A=\mathrm{M}_{n}(D)$ be as above. By [8, Th. 7.8], $\sigma$ is conjugate to some $\sigma^{\prime}$ of the form $\left(d_{i j}\right)_{i, j} \mapsto\left(d_{j i}^{\tau}\right)_{i, j}$ where $\tau: D \rightarrow D$ is an anti-automorphism, so assume $\sigma$ is in this form. This implies that $u$ commutes with the standard matrix units $\left\{e_{i j}\right\}$ (because they satisfy $e_{i j}^{\sigma \sigma}=e_{i j}$ ), hence we may view $u$ as an element of $D$ (embedded diagonally in $A=\mathrm{M}_{n}(D)$ ) which satisfies $d^{\tau \tau}=u d u^{-1}$ for all $d \in D$. Now, it is enough to show that $(D, \tau)$ can be made standard by conjugation.

Assume that there exists $v \in D^{\times}$with $u^{-1} v^{\tau}=v$. Then $u^{\prime}:=v\left(v^{\tau}\right)^{-1} u=1$, so by Proposition 2.4 (or by computation), $\tau^{\prime}: d \mapsto v d^{\tau} v^{-1}$ is an involution. Observe that $d \mapsto u^{-1} d^{\tau}$ is an involutary additive map, hence $v=d+u^{-1} d^{\tau}$ always satisfies $u^{-1} v^{\tau}=v$. If $D=E \times E^{\text {op }}$ with $E$ a division ring, take $d=\left(1_{E}, 0_{E}^{\text {op }}\right)$ to get $v \in D^{\times}$. Otherwise, any $d$ with $v=d+u^{-1} d^{\tau} \neq 0$ will do. If such $d$ does not exist, then $d^{\tau}=-u d$ for all $d \in D$. Taking $d=1$ implies $u=-1$ and hence $\tau=\operatorname{id}_{D}$. Thus, either $(u, \tau)$ can be conjugated to $\left(1, \tau^{\prime}\right)$ with $\tau^{\prime}: D \rightarrow D$ an involution, or $\tau=\mathrm{id}_{D}$ and $u=-1$ (in which case $D$ is a field). This implies (1) and (3).

It remains to check (2). Indeed, when $D$ is not a division ring, there is an isomorphism $D \cong E \times E^{\mathrm{op}}$ and under that isomorphism $\tau$ is given by $\left(a, b^{\mathrm{op}}\right)^{\tau}=$ $\left(t b t^{-1}, a^{\text {op }}\right)$ for some $t \in E$. Since $\tau$ is an involution, it must be the exchange involution.

Remark 2.7. Proposition 2.6 is the reason why many authors require $\sigma$ to be an involution in the definition of unitary rings. The author does not know if there 
exists a similar result for semilocal rings (i.e. a statement guaranteeing that $\sigma$ can always be conjugated into an involution). See [9, Rm. 7.7] for further discussion.

Proposition 2.8. Let $(A, \sigma, u, \Lambda)$ be a unitary ring such that $A$ is a semisimple (artinian) ring. Then $(A, \sigma, u, \Lambda)$ factors into a product

$$
(A, \sigma, u, \Lambda) \cong \prod_{i=1}^{t}\left(A_{i}, \sigma_{i}, u_{i}, \Lambda_{i}\right):=\left(\prod_{i} A_{i}, \prod_{i} \sigma,\left(u_{i}\right)_{i}, \prod_{i} \Lambda_{i}\right)
$$

with each $\left(A_{i}, \sigma_{i}, u_{i}, \Lambda_{i}\right)$ simple artinian.

Proof. See [20, p. 486], for instance.

§2.4. Orthogonality. We now define a notion of orthogonality for simple artinian unitary rings which will be used later in the text (compare with the orthogonality defined in [3, Ch. 4, §2] in the commutative case). This notion is used implicitly and repeatedly in $[20$.

Definition 2.9. A simple artinian unitary ring $(A, \sigma, u, \Lambda)$ is called orthogonal if:

(1) $A$ is simple and of finite dimension over its center, denoted $K$,

(2) $\left.\sigma\right|_{K}=\operatorname{id}_{K}$,

(3) $\Lambda$ is a K-vector space and $\operatorname{dim}_{K} \Lambda=\frac{1}{2} n(n-1)$ where $n=\sqrt{\operatorname{dim}_{K} A}$.

If in addition $A \cong \mathrm{M}_{n}(K)$ (i.e. $A$ is split as a central simple $K$-algebra), then we say that $(A, \sigma, u, \Lambda)$ is split-orthogonal.

Remark 2.10. We use the term "orthogonal" because isometry groups of unimodular quadratic forms over an orthogonal unitary ring $(A, \sigma, u, \Lambda)$ are forms of the the orthogonal group $\mathbf{O}_{m}(K)$, when viewed as algebraic groups over $K:=\operatorname{Cent}(A)$. This follows from the discussion in $\$ 5.1$ below. (A symplectic unitary ring can likewise be defined by replacing $\frac{1}{2} n(n-1)$ with $\frac{1}{2} n(n+1)$ in condition (3).)

Example 2.11. If $(A, \sigma, u, \Lambda)$ is simple artinian and in standard from (see $§ 2.3$ ), then it is split-orthogonal if and only if $A \cong \mathrm{M}_{n}(K)$ for a field $K, \sigma$ is the matrix transposition, $u=1$, and $\Lambda=\Lambda^{\mathrm{min}}$.

Generalizing the example, let $(A, \sigma, u, \Lambda)$ be a unitary ring such that $\sigma$ is an involution. If $(A, \sigma, u, \Lambda)$ satisfies conditions (1) and (2), then $A$ is a central simple algebra over its center $K$ (see [16, §1]) and $\sigma$ is an involution of the first kind (i.e. it fixes $\operatorname{Cent}(A))$. This easily implies $u \in\{ \pm 1\}$. By [16, Pr. 2.6], when char $K \neq 2$, there is $\varepsilon \in\{ \pm 1\}$ such that

$$
\operatorname{dim}_{K}\left\{a \pm a^{\sigma} \mid a \in A\right\}=\frac{1}{2} n(n \pm \varepsilon)
$$

where $n=\operatorname{deg} A:=\sqrt{\operatorname{dim}_{K} A}$. When $\varepsilon=1$ (resp. $\varepsilon=-1$ ) $\sigma$ is called orthogonal (resp. symplectic). Furthermore, when char $K=2$, we always have

$$
\operatorname{dim}_{K} \Lambda^{\min }=\operatorname{dim}_{K}\left\{a-a^{\sigma} \mid a \in A\right\}=\frac{1}{2} n(n-1) .
$$

Thus, when $\sigma$ is an involution, condition (3) is equivalent to having one of the following:

(3a) char $K \neq 2, \sigma$ is orthogonal and $u=1$,

(3b) char $K \neq 2, \sigma$ is symplectic and $u=-1$,

(3c) $\operatorname{char} K=2$ and $\Lambda=\Lambda^{\mathrm{min}}$. 
See [16, §2] for further details about orthogonal and symplectic involutions.

We further recall that the index of a central simple $K$-algebra $A$ admitting an involution of the first kind is a power of 2 ([16, Cr. 2.8]). Thus, if $\operatorname{deg} A$ is odd, then $A$ is split (i.e. ind $A=1$ ).

Proposition 2.12. Orthogonality (resp. split-orthogonality) of simple artinian unitary rings is preserved under conjugation (see Proposition 2.4). Furthermore, if $e \in A$ is an idempotent satisfying $e^{\sigma}=e$, then $(A, \sigma, u, \Lambda)$ is orthogonal (resp. splitorthogonal) if and only if (eAe, $\left.\left.\sigma\right|_{e A e}, u e, e \Lambda e\right)$ is orthogonal (resp. split-orthogonal).

Proof. That orthogonality (resp. split-orthogonality) is invariant under conjugation is clear from the definitions, so we turn to prove the second statement. Note that since $(A, \sigma, u, \Lambda)$ is simple and $e^{\sigma}=e$, we have $A e A=A$ (because $\left.(A e A)^{\sigma}=A e A\right)$. Morita Theory (see [18, $\S 18 \mathrm{D}]$, for instance) now implies that $A$ is simple if and only if $e A e$ is simple, and $\operatorname{Cent}(e A e)=e \operatorname{Cent}(A)$. Writing $K=\operatorname{Cent}(A)$, it follows that $A$ is a (split) central simple $K$-algebra if and only if $e A e$ is. Furthermore, in this case, it is easy to see that $\sigma$ is of the first kind if and only if $\left.\sigma\right|_{e A e}$ is. Therefore, we may assume $A$ is a central simple $K$-algebra and $\sigma$ is of the first kind.

We claim that $\Lambda$ is a $K$-vector space if and only if $e \Lambda e$ is a $K$-vector space. (In fact, this is clear when char $K \neq 2$ because $\Lambda=\Lambda^{\text {min }}$ and $\sigma$ is of the first kind.) One direction is evident so we turn to show the other. Assume $e \Lambda e$ is a $K$-vector space and let $a \in \Lambda$ and $k \in K$. Write $1_{A}=\sum_{i} x_{i} e y_{i}$ for $\left\{x_{i}, y_{i}\right\}_{i} \subseteq A$. Then $a=\left(\sum_{i} x_{i} e y_{i}\right)^{\sigma} a\left(\sum_{j} x_{j} e y_{j}\right)=\sum_{i, j} a_{i j}$ where $a_{i j}=y_{i}^{\sigma} e x_{i}^{\sigma} a x_{j} e y_{j}$. Observe that since $a^{\sigma} u=-a, a_{j i}=-a_{i j}^{\sigma} u$. Since $e \Lambda e$ is a $K$-vector space, $k \cdot e x_{i}^{\sigma} a x_{i} e \in e \Lambda e \subseteq \Lambda$ for all $i$, hence $k \cdot a_{i i}=y_{i}^{\sigma}\left(k e x_{i}^{\sigma} a x_{i} e\right) y \in \Lambda$. Now, $k a=\sum_{i, j} a_{i j}=k \sum_{i<j}\left(a_{i j}-\right.$ $\left.a_{i j}^{\sigma} u\right)+k \sum_{i} a_{i i}=\sum_{i<j}\left(k a_{i j}-\left(k a_{i j}\right)^{\sigma} u\right)+\sum_{i}\left(k a_{i i}\right) \in \Lambda$, as required.

Assume $\Lambda$ is a $K$-vector space. It is left to show that $\operatorname{dim}_{K} \Lambda=\frac{1}{2} n(n-1)$ if and only if $\operatorname{dim}_{K} e \Lambda e=\frac{1}{2} m(m-1)$, where $n=\operatorname{deg} A$ and $m=\operatorname{deg} e A e$. Observe that by the above discussion, when $\sigma$ is an involution, we always have $\operatorname{dim}_{K} \Lambda=$ $\frac{1}{2} n(n \pm 1)$ and $\operatorname{dim}_{K} e \Lambda e=\frac{1}{2} m(m \pm 1)$. Thus, by Proposition 2.6, the same holds for arbitrary $\sigma$. Let $f=1-e$. There is nothing to prove if $f=0$. Otherwise, $\operatorname{deg} f A f=n-m$, hence $\operatorname{dim}_{K} f \Lambda f=\frac{1}{2}(n-m)(n-m \pm 1)$. It is easy to check that $\operatorname{dim} \Lambda=\operatorname{dim} e \Lambda e+\operatorname{dim} f \Lambda f+\operatorname{dim} e A f=\operatorname{dim} e \Lambda e+\operatorname{dim} f \Lambda f+m(n-m)$, and this implies $\operatorname{dim} \Lambda=\frac{1}{2} n(n-1)$ if and only if $\operatorname{dim} e \Lambda e=\frac{1}{2} m(m-1)$.

§2.5. Semiperfect Rings. We finish this section with recalling several facts about semiperfect rings. Proofs and additional details can be found in [21, §2.7-§2.9].

A ring $A$ is called semiperfect if it satisfies the following equivalent conditions:

(a) $A$ is is semilocal and $\operatorname{Jac}(A)$ is idempotent lifting.

(b) All finitely generated right (or left) $A$-modules have a projective cover (see 21, Df. 2.8.31]).

(c) There exists orthogonal idempotents $e_{1}, \ldots, e_{n} \in A$ with $\sum_{i} e_{i}=1$ and such that $e_{i} A e_{i}$ is local for all $i$.

In this case, any system of orthogonal idempotents in $A / \operatorname{Jac}(A)$ can be lifted to a system of orthogonal idempotents in $A$. Furthermore, $e A e$ is semiperfect for any idempotent $e \in A$.

Examples of semiperfect rings include all one-sided artinian rings, and more gerenally, all semilocal rings $A$ with $A=\lim _{\longleftarrow}\left\{A / \operatorname{Jac}(A)^{n}\right\}_{n \in \mathbb{N}}$. Further examples that will be used later can be obtained from the following proposition. 
Proposition 2.13. Let $R$ be a henselian local (commutative) ring, and let $A$ be an $R$-algebra. Then $A$ is semiperfect if one of the following holds:

(1) $R$ is noetherian and $A$ is finitely generated as an $R$-module.

(2) $R$ is a valuation ring and $A$ is $R$-torsion-free and of finite rank over $R \mathbb{1}$

Proof. When (1) holds, this follows from [1, Th. 22] or [25, Lm. 12]. When (2) holds, $A$ is semilocal by [27, Th. 5.4]. Let $e \in A$ be an idempotent such that $e A e$ has no idempotents other than $e$ and 0 . The proof of [25, Lm. 14] then implies that $e A e$ is local. Replacing $A$ with $(1-e) A(1-e)$ and repeating this procedure yields a (finite) system of orthogonal idempotents $e=e_{1}, e_{2} \ldots, e_{t} \in A$ with $\sum_{i} e_{i}=1$ and such that $e_{i} A e_{i}$ local for all $i$, so $A$ is semiperfect.

Let $A$ be a semiperfect ring. Then, up to isomorphism, there exist finitely many indecomposable projective $A$-modules, $\left\{P_{i}\right\}_{i=1}^{t}$, and every $P \in \mathscr{P}(A)$ can be written as $P \cong \bigoplus_{i=1}^{t} P_{i}^{n_{i}}$ with $\left(n_{i}\right)_{i=1}^{t}$ uniquely determined. If $\bar{A}=A / \operatorname{Jac}(A)$, then $\left\{\bar{P}_{i}:=P_{i} / P_{i} \operatorname{Jac}(A)\right\}_{i=1}^{t}$ are the simple $\bar{A}$-modules, up to isomorphism.

The modules $P_{1}, \ldots, P_{t}$ can be constructed as follows: Write $\bar{A}$ as a product of simple artinian rings $\prod_{i=1}^{t} A_{i}$, let $\varepsilon_{i}$ be a primitive idempotent in $A_{i}$, and let $e_{i}$ be a lifting of $\varepsilon_{i}$ to $A$. Then $e_{1} A, \ldots, e_{t} A$ are the indecomposable projective right $A$-modules, up to isomorphism.

\section{Reflections And Quasi-Reflections}

In this section we introduce and study quasi-reflections, which slightly extend a notion of reflections used by Reiter [20. Throughout, $(A, \sigma, u, \Lambda)$ is a unitary ring, and $(P,[\beta])$ be a quadratic space over $(A, \sigma, u, \Lambda)$.

Let $e, f \in A$ be idempotents. An element $a \in e A f$ is called $(e, f)$-invertible if there exists $a^{\prime} \in f A e$ such that $a a^{\prime}=e$ and $a^{\prime} a=f$. It is easy to see that $a^{\prime}$ is unique and has $a$ as its $(f, e)$-inverse. We hence write $a^{\prime}=\left({ }_{e} a_{f}\right)^{\circ}$, or just $a^{\prime}=a^{\circ}$ when $e, f$ are understood from the context. Notice that there exists an $(e, f)$ invertible element if and only $e A \cong f A$, in which case we write $e \sim f$. Indeed, left multiplication by an $(e, f)$-invertible element gives an isomorphism from $f A$ to $e A$, and any isomorphism $f A \rightarrow e A$ is easily seen to be of this form.

Lemma 3.1. Let $e, f \in A$ be idempotents, and set $\bar{x}:=x+\operatorname{Jac}(A) \in A / \operatorname{Jac}(A)$ for all $x \in A$. Then $a \in e A f$ is $(e, f)$-invertible if and only if $\bar{a}$ is $(\bar{e}, \bar{f})$-invertible.

Proof. We only show the non-trivial direction. Assume $\bar{a}$ has an $(\bar{e}, \bar{f})$-inverse $\bar{b}$ with $b \in f A e$. Then $\overline{a b} \in(\overline{e A e})^{\times}$, hence $a b \in e A e^{\times}$. (This follows from the easy fact that $\operatorname{Jac}(A) \cap e A e=e \operatorname{Jac}(A) e=\operatorname{Jac}(e A e)$.) Likewise, $b a \in f A f^{\times}$. Let $c$ be the inverse of $a b$ in $e A e$, and let $d$ be the inverse of $b a$ in $f A f$. Then $a(b c)=e$, $(d b) a=f$, and $d b=(d b) e=(d b) a(b c)=f(b c)=b c$. Thus, $a^{\prime}=b c=d b$ is an $(e, f)$-inverse of $a$.

Let $e \in A$ be an idempotent, let $y \in P e$, and let $c \in e^{\sigma} \hat{\beta}(y) e=\beta(y, y)+e^{\sigma} \Lambda e$ be $\left(e^{\sigma}, e\right)$-invertible. We define $s_{y, e, c}: P \rightarrow P$ by

$$
s_{y, e, c}(x)=x-y \cdot c^{\circ} \cdot h_{\beta}(y, x) .
$$

\footnotetext{
${ }^{1}$ For an integral domain $R$, the rank an $R$-module $M$ is $\operatorname{dim}_{K} M \otimes_{R} K$, where $K$ is the fraction field of $R$.
} 
Observe that $s_{y, e, c}$ is completely determined by the class $[\beta]$. Following [20, we call $s_{y, e, c}$ an $e$-reflection. We will also use the name quasi-reflection, which does not restrict us to a particular idempotent $e$. A reflection of $(P,[\beta])$ is a 1-reflection. When we want to stress the quadratic form $[\beta]$, we shall write $s_{y, e, c}^{[\beta]}$ instead of $s_{y, e, c}$.

Remark 3.2. Reiter's definition of e-reflections ([20, Df. 1.2]) is essentially the same, except that he assumes $e=e^{\sigma}$ (in which case $c^{\circ}$ is just the inverse of $c$ in $e A e)$. The generalization defined here will play a crucial role later in the text.

Proposition 3.3. In the previous setting, $s_{y, e, c}$ is an isometry of $(P,[\beta])$. Its inverse is $s_{y, e, c^{\sigma} u}$.

Proof. This is similar to the proof of [20, Pr. 1.3]; replace the usual inverses with $\left(e^{\sigma}, e\right)$-inverses.

Lemma 3.4. Let $e, f \in A$ be idempotents.

(i) If $e \sim f$, then e-reflections and $f$-reflections coincide.

(ii) If ef $=f e=0$, then the composition of an e-reflection and an $f$-reflection is an $(e+f)$-reflection. Specifically, $s_{y, e, c} s_{z, f, d}=s_{y+z, e+f, c+d+h(y, z)}$.

Proof. (i) Let $a \in e A f$ be an $(e, f)$-invertible element. It is a straightforward computation to verify that $s_{y, e, c}=s_{y a, f, a^{\sigma} c a}$, which proves the claim.

(ii) Throughout the proof, we shall make repeated implicit usage of the fact that $y f=z e=0$ and $f c^{\circ}=c^{\circ} f^{\sigma}=e d^{\circ}=d^{\circ} e^{\sigma}=0$, which easily follows from ef $=f e=0$.

Observe first that

$$
\begin{aligned}
c+d+h(y, z) & \in e^{\sigma} \hat{\beta}(y) e+f^{\sigma} \hat{\beta}(z) f+e^{\sigma} h(y, z) f \\
& \subseteq(e+f)^{\sigma}(\hat{\beta}(z)+\hat{\beta}(y)+h(y, z))(e+f) \\
& =(e+f)^{\sigma} \hat{\beta}(z+y)(e+f)
\end{aligned}
$$

and that $c+d+h(y, z)$ is $\left((e+f)^{\sigma}, e+f\right)$-invertible with inverse $c^{\circ}+d^{\circ}-c^{\circ} h(y, z) d^{\circ}$. Thus, $r:=s_{y+z, e+f, c+d+h(y, z)}$ is an $(e+f)$-reflection. Now, for all $x \in P$, we have

$$
\begin{aligned}
r x & =x-(y+z)\left(c^{\circ}+d^{\circ}-c^{\circ} h(y, z) d^{\circ}\right) h(y+z, x) \\
& =x-\left(y c^{\circ}+z d^{\circ}-y c^{\circ} h(y, z) d^{\circ}\right)(h(y, x)+h(z, x)) \\
& =x-z d^{\circ} h(z, x)-y c^{\circ} h(y, x)+y c^{\circ} h(y, z) d^{\circ} h(z, x) \\
& =\left(x-z d^{\circ} h(z, x)\right)-y c^{\circ} h\left(y, x-z d^{\circ} h(z, x)\right) \\
& =s_{y, e, c}\left(s_{z, f, d} x\right),
\end{aligned}
$$

as required.

Lemma 3.5. Let $e \in A$ be an idempotent and let $x, y \in P e$.

(i) If $c:=h_{\beta}(x-y, x)$ is $\left(e^{\sigma}, e\right)$-invertible, then $s_{x-y, e, c}(x)=y$.

(ii) If there exist $z \in P e$ and $\left(e^{\sigma}, e\right)$-invertible $c \in \hat{\beta}(z)$ such that $d:=h_{\beta}(y, w)$ is $\left(e^{\sigma}, e\right)$-invertible for $w=y-s_{z, e, c}(x)=y-x+z c^{\circ} h_{\beta}(z, x)$, then $s_{w, e, d} s_{z, e, c}(x)=y$.

Proof. This is essentially the same as the proofs of Lemma 1.4 and Lemma 1.5 in 20]; replace the usual inverses with $\left(e^{\sigma}, e\right)$-inverses. 
Remark 3.6. (i) When applying conjugation (see Proposition 2.4) with respect to $v \in A^{\times}$, $e$-reflections remain $e$-reflections. Indeed, it is straightforward to check that $s_{y, e, c}^{[\beta]}=s_{y, e, v c}^{[v \beta]}$ (note that if $a^{\sigma^{\prime}}:=v a^{\sigma} v^{-1}$, then $\left.\left({ }_{e^{\sigma^{\prime}}} v c_{e}\right)^{\circ}=c^{\circ} v^{-1}\right)$. (In Reiter's setting, which assumes $e=e^{\sigma}$, e-reflections are preserved only when $e$ commutes with $v$, for otherwise, $e$ is not invariant under the conjugation of $\sigma$ by v.)

(ii) Let $e, f \in A$ be idempotents with $e=e^{\sigma}$ and $f \in e A e$. Then $e$-transfer (see Proposition [2.5) sends $f$-reflections of $(P,[\beta])$ to $f$-reflections of $\left(P e,\left[\beta_{e}\right]\right)$. Indeed, $\left.s_{y, e, c}^{[\beta]}\right|_{P e}=s_{y, e, c}^{\left[\beta_{e}\right]}$.

\section{Witt's Extension Theorem}

Using methods of Reiter 20 and the notion of quasi-reflections above, we now show that every isometry between subspaces of a unimodular quadratic space over a semiperfect ring can be extended to an isometry of the whole quadratic space. Furthermore, with small exception, the resulting isometry is a product of quasireflections. We compare our results with those of Reiter in Remark 4.12

$\S 4.1$. General Setting. We set some general notation that will be used throughout. Let $(A, \sigma, u, \Lambda)$ be a semiperfect unitary ring. For $P \in \mathscr{P}(A)$, set $\bar{P}:=$ $P / P \operatorname{Jac}(A)$. In particular, $\bar{A}=A / \operatorname{Jac}(A)$. We shall occasionally view $\bar{P}$ as a right $\bar{A}$-module. The image of $x \in P$ in $\bar{P}$ will be denoted by $\bar{x}$. Note that $P, Q \in \mathscr{P}(A)$ are isomorphic if and only if $\bar{P} \cong \bar{Q}$, because $P$ is a projective cover of $\bar{P}$ and projective covers are unique up to isomorphism.

Let $\bar{\Lambda}=\{\bar{\lambda} \mid \lambda \in \Lambda\}$ and let $\bar{\sigma}$ be the map induced by $\sigma$ on $\bar{A}$. Then $(\bar{A}, \bar{\sigma}, \bar{u}, \bar{\Lambda})$ is a semisimple unitary ring, hence, by Proposition 2.8 it factors into a product

$$
(\bar{A}, \bar{\sigma}, \bar{u}, \bar{\Lambda}) \cong \prod_{i=1}^{\ell}\left(A_{i}, \sigma_{i}, u_{i}, \Lambda_{i}\right)
$$

with each $\left(A_{i}, \sigma_{i}, u_{i}, \Lambda_{i}\right)$ a simple artinian unitary ring (see $\S 2.3$. We write $A_{i}=$ $\mathrm{M}_{n_{i}}\left(D_{i}\right)$ with $D_{i}$ a division ring, or a product of a division ring and its opposite.

By Proposition 2.6. for every $1 \leq i \leq \ell$, there exists $v_{i} \in A_{i}^{\times}$such that the conjugation (see Proposition 2.4) of $\left(\sigma_{i}, u_{i}, \Lambda_{i}\right)$ by $v_{i}$ is standard (see $\$ 2.3$ ). Choose $v \in A^{\times}$whose image in $A_{i}$ is $v_{i}$. Then, by conjugating $(\sigma, u, \Lambda)$ with $v$, we may assume that $\left(A_{i}, \sigma_{i}, u_{i}, \Lambda_{i}\right)$ is in standard form for all $i$. Note that conjugation preserves quasi-reflections by Remark 3.6(i), so this is allowed if our goal is to prove that certain isometries extend to a product of quasi-reflections.

Now, let $\varepsilon_{i}$ denote the standard matrix unit $e_{11}$ in $A_{i}$. Then $\varepsilon_{1}, \ldots, \varepsilon_{\ell}$ are orthogonal $\bar{\sigma}$-invariant idempotents. Since $\operatorname{Jac}(A)$ is idempotent lifting, we can lift $\varepsilon_{1}, \ldots, \varepsilon_{\ell}$ to orthogonal idempotents $e_{1}, \ldots, e_{\ell} \in A$. The idempotents $e_{i}$ may not be invariant under $\sigma$, but we have $e_{i} A \cong e_{i}^{\sigma} A$ as right $A$-modules (because $\overline{e_{i} A}=\overline{e_{i}^{\sigma} A}$ ), and hence $e_{i} \sim e_{i}^{\sigma}$.

Next, we set

$$
\left(A_{(i)}, \sigma_{(i)}, u_{(i)}, \Lambda_{(i)}\right)=\left(\varepsilon_{i} A_{i} \varepsilon_{i},\left.\sigma_{i}\right|_{\varepsilon_{i} A_{i} \varepsilon_{i}}, \varepsilon_{i} u_{i}, \varepsilon_{i} \Lambda_{i} \varepsilon_{i}\right) .
$$

Note that by Proposition 2.12, $\left(A_{i}, \sigma_{i}, u_{i}, \Lambda_{i}\right)$ is split-orthogonal if and only if $\left(A_{(i)}, \sigma_{(i)}, u_{(i)}, \Lambda_{(i)}\right)$ is split-orthogonal, and in this case, $A_{(i)}$ is a field, $\sigma_{(i)}=\mathrm{id}_{A_{(i)}}$, $u_{(i)}=1$ and $\Lambda_{(i)}=0$. Also note that $A_{(i)} \cong D_{i}$. 
Let $(P,[\beta])$ be a quadratic space over $(A, \sigma, u, \Lambda)$. Then $(P,[\beta])$ gives rise to a quadratic space $(\bar{P},[\bar{\beta}])$ over $(\bar{A}, \bar{\sigma}, \bar{u}, \bar{\Lambda})$; the map $\bar{\beta}$ is defined by

$$
\bar{\beta}(\bar{x}, \bar{y})=\overline{\beta(x, y)} \quad \forall x, y \in P .
$$

Since $(\bar{A}, \bar{\sigma}, \bar{u}, \bar{\Lambda})$ factors into a product of unitary rings, the datum of $(\bar{P},[\bar{\beta}])$ is equivalent to the datum of quadratic spaces $\left(P_{i},\left[\beta_{i}\right]\right)_{i=1}^{\ell}$ over $\left(A_{i}, \sigma_{i}, u_{i}, \Lambda_{i}\right)_{i=1}^{\ell}$. Specifically, if we write $\bar{P}=\prod_{i=1}^{\ell} P_{i}$ with $P_{i}$ a right $A_{i}$-module, then $\beta_{i}$ is just the restriction of $\beta$ to the copy of $P_{i}$ in $\bar{P}$. We further set $P_{(i)}=P_{i} \varepsilon_{i} \in \mathscr{P}\left(A_{(i)}\right)$ and let $\beta_{(i)}=\left(\beta_{i}\right)_{\varepsilon_{i}}:=\left.\beta_{i}\right|_{P_{(i)} \times P_{(i)}}$. Recall from Proposition 2.5 that $\left(P_{(i)},\left[\beta_{(i)}\right]\right)$ is a quadratic space over $\left(A_{(i)}, \sigma_{(i)}, u_{(i)}, \Lambda_{(i)}\right)$, which is the $\varepsilon_{i}$-transfer of $\left(P_{i}, \beta_{i}\right)$. As a result, we have

Next, let

$$
\left[\beta_{i}\right]=[0] \quad \Longleftrightarrow \quad\left[\beta_{(i)}\right]=[0] .
$$

$$
\pi_{i}: P \rightarrow P_{i}
$$

denote the map sending $x \in P$ to its image in $P_{i}$. We clearly have $\pi_{i}\left(P e_{i}\right)=P_{(i)}$ and $\pi_{i} \beta(x, y)=\beta_{i}\left(\pi_{i} x, \pi_{i} y\right)$.

Keeping the previous setting, let $Q \subseteq P$ be a submodule. Then $h_{\beta}$ induces a map

$$
L_{Q}=L_{[\beta], Q}: P \rightarrow Q^{*}:=\operatorname{Hom}_{A}(Q, A)
$$

sending $x \in P$ to $\left[y \mapsto h_{\beta}(x, y)\right] \in Q^{*}$. This map is evidently onto when $(P,[\beta])$ is unimodular and $Q$ is a summand of $P$. Lastly, set

$$
Q^{\perp}=Q^{\perp[\beta]}=\left\{x \in P: h_{\beta}(x, P)=0\right\},
$$

and let $\left.\beta\right|_{Q}$ denote the restriction of $\beta$ to $Q \times Q$.

$\S 4.2$. Lemmas. Before giving the main result, we collect several lemmas.

Lemma 4.1. Let $P \in \mathscr{P}(A)$. Then $P \cong P^{*} \Longleftrightarrow P$ is isomorphic to a direct sum of copies of the modules $e_{1} A, \ldots, e_{\ell} A$. (See §2.1 for the definition of $P^{*}$.)

Proof. It is straightforward to check that $\left(e_{i} A\right)^{*} \cong e_{i}^{\sigma} A$, and since $e_{i} \sim e_{i}^{\sigma}$, we have $e_{i}^{\sigma} A \cong e_{i} A$. This settles the direction "£", so we turn to show the converse.

Without loss of generality, $D_{i}$ is a division ring for $i \leq t$, and a product of a division ring and its opposite otherwise. In the latter case, $\varepsilon_{i}$ can be written as a sum of two orthogonal idempotents $\varepsilon_{i}=\delta_{i}+\delta_{i}^{\sigma}$. Now, $\varepsilon_{1}, \ldots, \varepsilon_{t}, \delta_{t+1}, \ldots, \delta_{\ell}, \delta_{t+1}^{\sigma}, \ldots, \delta_{\ell}^{\sigma}$ is a system of orthogonal idempotents in $\bar{A}$, hence it can be lifted to a system of orthogonal idempotents $f_{1}, \ldots, f_{2 \ell-t}$ in $A$. Moreover, $f_{1} A_{1}, \ldots, f_{2 \ell-t} A$ are the indecomposable projective right $A$-modules, up to isomorphism (see $\$ 2.5$ ).

Assume that $P \cong P^{*}$. Then $P \cong \bigoplus f_{i} A_{i}^{n_{i}}$ with $\left(n_{i}\right)_{i=1}^{2 \ell-t}$ uniquely determined. It is straightforward to check that $\left(f_{i} A_{i}\right)^{*} \cong f_{i}^{\sigma} A_{i}$. By the way we have constructed $f_{1}, \ldots, f_{2 \ell-t}$, we have $f_{i}^{\sigma} \sim f_{i}$ if $i \leq t$, and $f_{i}^{\sigma} \sim f_{i+(\ell-t)}$ if $t<i \leq \ell$. Thus, $n_{i}=n_{i+(\ell-t)}$ for all $t<i \leq \ell$. Since $e_{i} A \cong f_{i} A \oplus f_{i+(\ell-t)} A$ for $t<i \leq \ell$, it follows that $P \cong \bigoplus_{i=1}^{\ell}\left(e_{i} A\right)^{n_{i}}$, as required.

Lemma 4.2. Let $1 \leq i \leq \ell$, let $(P,[\beta])$ be a quadratic space, and let $Q, V \subseteq P$ summands of $P$. Then:

(i) $L_{[\beta], Q}(V)=Q^{*} \Longrightarrow L_{\left[\beta_{(i)}\right], Q_{(i)}}\left(V_{(i)}\right)=Q_{(i)}^{*}$.

(ii) $[\beta]$ is unimodular $\Longrightarrow\left[\beta_{i}\right]$ and $\left[\beta_{(i)}\right]$ are unimodular.

(iii) $V_{i}^{\perp\left[\beta_{i}\right]}=V_{(i)}^{\perp\left[\beta_{i}\right]}$ and $V_{i}^{\perp\left[\beta_{i}\right]} \varepsilon_{i}=V_{(i)}^{\perp\left[\beta_{(i)}\right]}$. 
$\left(\right.$ Here, $Q_{(i)}=\pi_{i}(Q) \varepsilon_{i}$ and $\left.V_{(i)}=\pi_{i}(V) \varepsilon_{i}.\right)$

Proof. (i) Let $\psi \in \operatorname{Hom}_{A_{(i)}}\left(Q_{(i)}, A_{(i)}\right)=\operatorname{Hom}_{\varepsilon_{i} A_{i} \varepsilon_{i}}\left(Q_{i} \varepsilon_{i}, \varepsilon_{i} A \varepsilon_{i}\right)$. As explained in the proof of Proposition [2.5, the functor $U \mapsto U \varepsilon_{i}: \mathscr{P}\left(A_{i}\right) \rightarrow \mathscr{P}\left(\varepsilon_{i} A_{i} \varepsilon_{i}\right)$ is an equivalence, hence there exists unique $\psi^{\prime} \in \operatorname{Hom}_{A_{i}}\left(Q_{i}, \varepsilon_{i} A_{i}\right)$ with $\left.\psi^{\prime}\right|_{Q_{(i)}}=\psi$. Since $Q$ is projective, there is $\varphi \in \operatorname{Hom}_{A}\left(Q, e_{i}^{\sigma} A\right)$ with $\pi_{i} \circ \varphi=\psi^{\prime} \circ \pi_{i}$. By assumption, there is $x \in V$ such that $L_{[\beta], Q}(x)=\varphi$, and by replacing $x$ with $x e_{i}$ we may assume $x=x e_{i} \in V_{i} e_{i}$. Now, for all $y \in Q e_{i}$, we have $L_{\left[\beta_{(i)}\right], Q_{(i)}}\left(\pi_{i} x\right)\left(\pi_{i} y\right)=$ $\beta_{(i)}\left(\pi_{i} x, \pi_{i} y\right)=\beta_{i}\left(\pi_{i} x, \pi_{i} y\right)=\pi_{i}(\beta(x, y))=\pi_{i}\left(L_{[\beta], Q}(x)(y)\right)=\pi_{i}(\varphi y)=\psi\left(\pi_{i} y\right)$, so $L_{\left[\beta_{(i)}\right], Q_{(i)}}\left(\pi_{i} x\right)=\psi$.

(ii) Putting $Q=V=P$ in (i) implies that $L_{\left[\beta_{(i)}\right]}$ is onto. Since $P_{(i)}^{*}$ and $P_{(i)}$ have the same length, $L_{\left[\beta_{(i)}\right]}$ is also injective, so $\left[\beta_{(i)}\right]$ is unimodular. By Proposition 2.5. $[\beta]$ is also unimodular.

(iii) This is routine. Use the fact that $V_{(i)} A_{i}=V_{i} A_{i} \varepsilon_{i} A_{i}=V_{i} A_{i}=V_{i}$.

Lemma 4.3. Let $P \in \mathscr{P}(A)$, and assume $P^{*}=U \oplus U^{\prime}$. Then $P$ factors as a direct sum $Q \oplus Q^{\prime}$ such that $U=\left\{f \in P^{*}: f\left(Q^{\prime}\right)=0\right\}$ and $U^{\prime}=\left\{f \in P^{*}: f(Q)=0\right\}$.

Proof. Let $\omega=\omega_{P}: P \rightarrow P^{* *}$ (see $\S 2.1$ for the definition). We identify $P^{* *}$ with $U^{*} \oplus U^{\prime *}$ via $g \mapsto\left(\left.g\right|_{U},\left.g\right|_{U^{\prime}}\right)$. Let $Q=\omega^{-1}\left(U^{*}\right)$ and $Q^{\prime}=\omega^{-1}\left(U^{\prime *}\right)$. We clearly have $P=Q \oplus Q^{\prime}$. Furthermore, for $f \in P^{*}$, we have $f \in U \Longleftrightarrow \psi f=0$ for all $\psi \in U^{\prime *} \Longleftrightarrow(\omega x) f=0$ for all $x \in Q^{\prime} \Longleftrightarrow(f x)^{\sigma} u=0$ for all $x \in Q^{\prime}$ $\Longleftrightarrow f x=0$ for all $x \in Q^{\prime}$. Thus, $U=\left\{f \in P^{*}: f\left(Q^{\prime}\right)=0\right\}$, and likewise, $U^{\prime}=\left\{f \in P^{*}: f(Q)=0\right\}$.

Lemma 4.4. Assume $A \cong \mathbb{F}_{2} \times \mathbb{F}_{2}$ and $\sigma$ is the exchange involution (this forces $u=1$ and $\Lambda=\{0,1\})$. Let $(P,[\beta])$ be a quadratic space over $A$, let $V \subseteq P$ be a submodule, and let $x \in P, z \in V$ be such that $h_{\beta}(x, z)=1$. Then the following conditions are equivalent:

(a) There is no $z^{\prime} \in V$ with $h_{\beta}\left(x, z^{\prime}\right)=1$ and $\hat{\beta}\left(z^{\prime}\right)=\Lambda=\{0,1\}$.

(b) $h_{\beta}(z, z)=1$ and $\left[\left.\beta\right|_{z^{\perp} \cap V}\right]=[0]$.

In this case, $L_{V}(V) \cong A$.

Proof. For the equivalence (a) $\Longleftrightarrow(\mathrm{b})$, see [20, Lm. 3.8c]. (In Reiter's notation, $H_{f}=H \cap f^{\perp}$ and $N$ stands for the zero form [0].) When (b) holds, we have $V=z A \oplus\left(z^{\perp} \cap V\right)$ since $x=\left(z h_{\beta}(z, x)\right)+\left(x-z h_{\beta}(z, x)\right)$ for all $x \in V$. This means that $L_{V}(V)=L_{V}(z A)$, which is easily seen to imply $L_{V}(V) \cong(z A)^{*} \cong A^{*} \cong A$.

$\S 4.3$. Witt's Extension Theorem. We are now in position to phrase and prove an analogue of Witt's Extension Theorem (Theorems 4.5 and 4.8). Following are several immediate (and less technical) corollaries. We compare our results with those of Reiter [20 in Remark 4.12 below.

Theorem 4.5. Let $(P,[\beta])$ be a quadratic space, let $Q, S, V$ be summands of $P$, and let $\psi:\left(Q,\left[\left.\beta\right|_{Q}\right]\right) \rightarrow\left(S,\left[\left.\beta\right|_{S}\right]\right)$ be an isometry. Assume that the following holds:

(1a) $L_{Q}(V)=Q^{*}$ and $L_{S}(V)=S^{*}$.

(1b) $\psi x-x \in V$ for all $x \in Q$.

(1c) $Q \cong Q^{*}$ and $S \cong S^{*}$.

(2a) If $\left(A_{i}, \sigma_{i}, u_{i}, \Lambda_{i}\right)$ is split-orthogonal, then $Q_{i}=0$ or $\left[\left.\beta_{i}\right|_{V_{i}}\right] \neq 0$.

(2b) If $D_{i} \cong \mathbb{F}_{2}$ and $\left(A_{i}, \sigma_{i}, u_{i}, \Lambda_{i}\right)$ is split-orthogonal, then $\left[\left.\beta_{i}\right|_{V_{i} \cap V_{i}^{\perp}}\right] \neq[0]$. 
(2c) If $D_{i} \cong \mathbb{F}_{2} \times \mathbb{F}_{2}$, then there is no $z \in V_{(i)}=V_{i} e_{i}$ satisfying $h_{\beta_{i}}(z, z)=\varepsilon_{i}$ and $\left[\left.\beta_{i}\right|_{z^{\perp} \cap V_{i}}\right]=[0]$.

Then there is $\varphi \in O(P,[\beta])$ such that $\varphi$ is a product of quasi-reflections taken with respect to elements of $V$, and $\left.\varphi\right|_{Q}=\psi$. (The former implies $\varphi x-x \in V$ for all $x \in P$.)

We first prove the following special case.

Lemma 4.6. Theorem 4.5 holds when $Q \cong e_{i} A$ for some $1 \leq i \leq \ell$. In fact, in this case, $\varphi$ can be taken to be a product of $e_{i}$-reflections.

Proof. The proof is based on reduction to the proof of [20, Th. 4.1], with certain modifications (particularly in the case $D_{i} \cong \mathbb{F}_{2} \times \mathbb{F}_{2}$ ). Throughout, $h=h_{\beta}$.

Write $e=e_{i}$. Since $Q \cong e A$, there is $x \in Q$ with $x e=x$ and $Q=x A$. Let $y=\psi x$. It is enough to find a product of $e$-reflections of $(P,[\beta])$, taken with respect to elements of $V$, that sends $x$ to $y$. We shall prove this by applying Lemma 3.5. except maybe in the case $D_{i} \cong \mathbb{F}_{2} \times \mathbb{F}_{2}$.

If $h(x-y, x)$ is $\left(e^{\sigma}, e\right)$-invertible, then $s_{x-y, e, h(x-y, x)}(x)=y($ and $x-y=x-\psi x \in$ $V$ by (1b)). If not, then it is enough to find $z \in V e_{i}$ and $\left(e^{\sigma}, e\right)$-invertible $c \in \hat{\beta}(z)$ such that

$$
\Phi(z, c):=h\left(y, y-x+z c^{\circ} h(z, x)\right)=h(y, y-x)+h(y, z) c^{\circ} h(z, x)
$$

is $\left(e^{\sigma}, e\right)$-invertible, in which case we shall have $s_{w, e, \Phi(z, c)} s_{z, e, c}(x)=y$ for $w=$ $(x-y)+z c^{\circ} h(z, x) \in V e_{i}$. Note that

$$
h(x-y, x)=h(x, x)-h(y, x)=h(y, y)-h(y, x)=h(y, y-x),
$$

so we may henceforth assume that $h(y, y-x)$ is not $\left(e^{\sigma}, e\right)$-invertible.

Reducing everything modulo $\operatorname{Jac}(A)$ (using Lemma 3.1), we see that it is enough to find $\bar{z} \in V_{(i)}=\overline{V e_{i}}$ and $\bar{c} \in \hat{\beta}_{(i)}(\bar{z}) \cap A_{(i)}^{\times}$such that $\bar{\Phi}(\bar{z}, \bar{c})$ is invertible in $A_{(i)}$, where

$$
\bar{\Phi}(\bar{z}, \bar{c})=h_{\beta_{(i)}}(\bar{y}, \bar{y}-\bar{x})+h_{\beta_{(i)}}(\bar{y}, \bar{z}) \bar{c}^{\circ} h_{\beta_{(i)}}(\bar{z}, \bar{x}) .
$$

We may assume that $h_{\beta_{(i)}}(\bar{y}, \bar{y}-\bar{x})$ is not invertible in $A_{(i)}$.

Now, conditions (1a) and (2a)-(2c) imply (respectively) that:

$(1 \mathrm{a}-i) L_{Q_{(i)}}\left(V_{(i)}\right)=Q_{(i)}^{*}$ and $L_{S_{(i)}}\left(V_{(i)}\right)=S_{(i)}^{*}$.

(2a-i) If $\left(A_{(i)}, \sigma_{(i)}, u_{(i)}, \Lambda_{(i)}\right)$ is split-orthogonal, then $\left[\left.\beta_{(i)}\right|_{V}\right] \neq 0$.

(2b-i) If $A_{(i)} \cong \mathbb{F}_{2}$ and $\left(A_{(i)}, \sigma_{(i)}, u_{(i)}, \Lambda_{(i)}\right)$ is split-orthogonal, then $\left[\left.\beta_{(i)}\right|_{V_{(i)} \cap V_{(i)}^{\perp}}\right] \neq$ $[0]$.

(2c-i) If $A_{(i)} \cong \mathbb{F}_{2} \times \mathbb{F}_{2}$, then there is no $\bar{z} \in V_{(i)}$ with $h_{\beta_{(i)}}(\bar{z}, \bar{z})=1$ and $\left[\left.\beta_{(i)}\right|_{z^{\perp} \cap V_{(i)}}\right]=[0]$.

(Use Lemma 4.2 and Proposition 2.12) We are now reduced to the setting of steps $2-5$ in the proof of $\left[20\right.$, Th. 4.1] (applied to $\left(P_{(i)},\left[\beta_{(i)}\right]\right)$ and $\left.\left(A_{(i)}, \sigma_{(i)}, u_{(i)}, \Lambda_{(i)}\right)\right)$, in which the existence of $\bar{z}$ and $\bar{c}$ as above is shown, except maybe in the case $A_{(i)} \cong \mathbb{F}_{2} \times \mathbb{F}_{2}$.

Assume henceforth that $A_{(i)} \cong \mathbb{F}_{2} \times \mathbb{F}_{2}$ (which means $\sigma_{(i)}$ is the exchange involution). Let $\varepsilon:=\varepsilon_{i}=1_{A_{(i)}}$. It is shown in cases 2 and 4 of the proof of [20, Th. 4.1], that either there are $\bar{z}$ and $\bar{c}$ as above, or there are $f^{\prime}, g^{\prime} \in V_{(i)}$ with $\varepsilon \in \hat{\beta}_{(i)}\left(f^{\prime}\right)$ and $\varepsilon \in \hat{\beta}_{(i)}\left(g^{\prime}\right)$ such that $s_{f^{\prime}, \varepsilon, \varepsilon}^{\left[\beta_{(i)}\right]} s_{g^{\prime}, \varepsilon, \varepsilon}^{\left[\beta_{(i)}\right]}(\bar{x})=\bar{y}$. In the former case we are done. In the latter case, let $f, g \in V e_{i}$ be such that $\bar{f}=f^{\prime}$ and $\bar{g}=g^{\prime}$, and choose $c \in e^{\sigma} \hat{\beta}(f) e$ 
and $d \in e^{\sigma} \hat{\beta}(g) e$ with $\bar{c}=\bar{d}=\varepsilon$. Then $c$ and $d$ are $\left(e^{\sigma}, e\right)$-invertible (Lemma 3.1), and $\pi_{i} s_{f, e, c}^{[\beta]} s_{g, e, d}^{[\beta]}(x)=\pi_{i} y$. Replacing $x$ with $s_{f, e, c}^{[\beta]} s_{g, e, d}^{[\beta]}(x)$, we may assume $\bar{x}=\bar{y}$. Under this new assumption, we establish the existence of $\bar{z}$ and $\bar{c}$ as above. Indeed, this amounts to finding $\bar{z} \in V_{(i)}$ with $h_{\beta_{(i)}}(\bar{x}, \bar{z})=1$ and $\hat{\beta}_{(i)}(\bar{z})=\Lambda_{(i)}=\{0,1\}$. By [20, Lm. 3.5], there is $\overline{z^{\prime}} \in V_{(i)}$ with $h\left(\bar{x}, \overline{z^{\prime}}\right)=1$. Condition (2c-i) and Lemma 4.4 now give there required $\bar{z} \in V_{(i)}$.

Proof of Theorem 4.5. The proof is based on the inductive step of [20, Th. 4.1] with certain modifications.

Step 1. If $Q=0$ there is nothing to prove. Otherwise, by Lemma 4.1, $e_{i} A$ is isomorphic to a summand of $Q$ for some $i$. Since $L_{Q}: V \rightarrow Q^{*}$ is onto and $Q^{*}$ is a projective right $A$-module, we can write $V=W \oplus R$ such that $\left.L_{Q}\right|_{W}$ is an isomorphism and $L_{Q}(R)=0$. Evidently, $\left(e_{i} A\right)^{*} \cong e_{i}^{\sigma} A \cong e_{i} A$ is also isomorphic to a summand $V^{\prime}$ of $W$. If $\left(A_{i}, \sigma_{i}, u_{i}, \Lambda_{i}\right)$ is not split-orthogonal or $D_{i} \not \mathbb{F}_{2} \times \mathbb{F}_{2}$, we choose $V^{\prime}$ arbitrarily. Otherwise, we claim that $V^{\prime}$ can be chosen such that condition (2a), (2b) and (2c) are satisfied when $V$ is replaced with $V^{\prime} \oplus R$.

Indeed, assume $\left(A_{i}, \sigma_{i}, u_{i}, \Lambda_{i}\right)$ is split-orthogonal and $D_{i} \not \mathbb{F}_{2}$. By condition (2a), we have $\left[\beta_{(i)}\right] \neq[0]$. Choose some $x \in V_{(i)}$ with $\hat{\beta}_{(i)}(x) \neq \Lambda_{(i)}=0$, and write $x=w+r$ with $w \in W_{(i)}, r \in R_{(i)}$. If $w=0$, then $\left[\beta_{(i)} \mid R_{(i)}\right] \neq[0]$, as required. Otherwise, $w \bar{A}$ is a summand of $\bar{W}$ (because $\bar{A}$ is semisimple) having $e_{i} A$ as projective cover. Thus, $W$ has a summand $V^{\prime} \cong e_{i} A$ whose image in $\bar{W}$ is $w A$. That $V^{\prime}$ satisfies $\left[\left.\beta_{(i)}\right|_{V_{(i)}^{\prime} \oplus R_{(i)}}\right] \neq[0]$, as required.

The same strategy also works when $D_{i} \cong \mathbb{F}_{2}$ by Lemma 4.2(iii); start with $x \in V_{(i)}^{\perp} \cap V_{(i)}=\left(V_{i}^{\perp} \cap V_{i}\right) \varepsilon_{i}$ such that $\hat{\beta}_{(i)}(x) \neq 0$.

When $D_{i} \cong \mathbb{F}_{2} \times \mathbb{F}_{2}$, by Lemma 4.4 and conditions (2c) and (1a), there is $z \in V_{(i)}$ and $x \in Q_{(i)}$ such that $h_{\beta_{(i)}}(x, z)=\varepsilon_{i}$ and $\hat{\beta}_{(i)}(z)=\Lambda_{(i)}=\left\{0, \varepsilon_{i}\right\}$. Write $z=w+r$ with $w \in W_{(i)}, r \in R_{(i)}$, and choose $V^{\prime}$ as above. (We must have $w \bar{A} \cong \varepsilon_{i} \bar{A}$. Otherwise, there is an idempotent $\delta \in A_{(i)} \cong \mathbb{F}_{2} \times \mathbb{F}_{2}$ such that $\delta \neq \varepsilon_{i}$ and $w \delta=w$, which implies $\varepsilon_{i}=h_{\beta_{(i)}}(x, z)=h_{\beta_{(i)}}(w+r, z)=h_{\beta_{(i)}}(w, z)=\delta^{\sigma} h_{\beta_{(i)}}(w, z) \in$ $\delta^{\sigma} A_{(i)}$, a contradiction.) Now, we have $z \in V_{(i)}^{\prime} \oplus R_{(i)}$ such that $\hat{\beta}_{(i)}(z)=\left\{0, \varepsilon_{i}\right\}$ and $h_{\beta_{(i)}}(x, z)=\varepsilon_{i}$, so condition (2c) holds for $V^{\prime} \oplus R$ by Lemma 4.4. This settles our claim about the choice of $V^{\prime}$.

Step 2. Write $W=V^{\prime} \oplus V^{\prime \prime}$, and let $U^{\prime}=L_{Q}\left(V^{\prime}\right)$ and $U^{\prime \prime}=L_{Q}\left(V^{\prime \prime}\right)$. Then $Q^{*}=U^{\prime} \oplus U^{\prime}$, so by Lemma 4.3, we have a decomposition $Q=Q^{\prime} \oplus Q^{\prime \prime}$ such that $L_{Q}\left(V^{\prime}\right)=\left\{f \in Q^{*}: f\left(Q^{\prime \prime}\right)=0\right\}=Q^{\prime *}$ and $L_{Q}\left(V^{\prime \prime}\right)=\left\{f \in Q^{*}: f\left(Q^{\prime}\right)=\right.$ $0\}=Q^{\prime \prime *}$. Now, $Q^{\prime *} \cong V^{\prime} \cong e_{i} A$, hence $Q^{\prime} \cong Q^{\prime * *} \cong\left(e_{i} A\right)^{*} \cong e_{i} A$, so $Q^{\prime} \cong Q^{\prime *}$. Thus, $Q^{\prime} \oplus Q^{\prime \prime *} \cong\left(Q^{\prime} \oplus Q^{\prime \prime}\right)^{*}=Q^{*} \cong Q \cong Q^{\prime} \oplus Q^{\prime \prime}$, which implies $Q^{\prime \prime *} \cong Q^{\prime \prime}$ (see the discussion at the end of $\S 2.5)$. Thus, we may apply induction to $\left(Q^{\prime \prime},\left[\left.\beta\right|_{Q^{\prime \prime}}\right]\right)$ and $\left.\psi\right|_{Q^{\prime \prime}}$. This yields $\varphi \in O(P,[\beta])$, a product of reflections taken with respect to elements of $V$, such that $\left.\varphi\right|_{Q^{\prime \prime}}=\left.\psi\right|_{Q^{\prime \prime}}$.

Step 3. We now claim that conditions (1a)-(1c) and (2a)-(2c) hold for the isometry $\left.\varphi^{-1} \psi\right|_{Q}: Q^{\prime} \rightarrow \varphi^{-1} \psi\left(Q^{\prime}\right)$ and the module $V^{\prime} \oplus R$ (in place of $\left.V\right)$. Indeed, (2a)-(2c) hold by our choice of $V^{\prime}$ (cf. Step 1), and (1c) was verified in Step 2. 
Next, notice that by construction, an element $z \in V$ lives in $V^{\prime} \oplus R$ if and only if $L_{Q}(z) \in Q^{\prime *}$, i.e. $0=L_{Q}(z)\left(Q^{\prime \prime}\right)=h\left(z, Q^{\prime \prime}\right)$. Now, for all $y \in Q^{\prime \prime}$, we have

$$
\begin{aligned}
h\left(\varphi^{-1} \psi x-x, y\right) & =h\left(\varphi^{-1} \psi x, y\right)-h(x, y)=h(\psi x, \varphi y)-h(x, y) \\
& =h(\psi x, \psi y)-h(x, y)=h(x, y)-h(x, y)=0 .
\end{aligned}
$$

This implies that $\varphi^{-1} \psi x-x \in V^{\prime} \oplus R$ for all $x \in Q^{\prime}$, so (1b) holds.

Finally, we have $L_{Q^{\prime}}\left(V^{\prime} \oplus R\right)=Q^{\prime *}$ by construction, so to show (1a) amounts to showing $L_{\varphi^{-1} \psi Q^{\prime}}\left(V^{\prime} \oplus R\right)=\left(\varphi^{-1} \psi Q^{\prime}\right)^{*}$. Let $f \in\left(\varphi^{-1} \psi Q^{\prime}\right)^{*}$. We extend $f$ to $\varphi^{-1} S=\varphi^{-1} \psi Q=\varphi^{-1} \psi Q^{\prime} \oplus \varphi^{-1} \psi Q^{\prime \prime}=\varphi^{-1} \psi Q^{\prime} \oplus Q^{\prime \prime}$ by setting it to be 0 on $Q^{\prime \prime}$. View $f \varphi^{-1}$ as an element of $S^{*}$. By (1a) (for $Q, S, V$ ), there is $x \in V$ with $L_{S}(x)=f \varphi^{-1}$. Now, for all $y \in \varphi^{-1} \psi Q$, we have $h\left(\varphi^{-1} x, y\right)=h(x, \varphi y)=$ $L_{S}(x)(\varphi y)=f \varphi^{-1} \varphi y=f y$, so $L_{\varphi^{-1} \psi Q^{\prime}}\left(\varphi^{-1} x\right)=f$. Since $\varphi$ is a product of reflections taken with respect to element of $V, \varphi(V)=V$, hence $\varphi^{-1} x \in V$. In addition, when $y \in Q^{\prime \prime}$, we have $h\left(\varphi^{-1} x, y\right)=f y=0$, so $\varphi^{-1} x \in V^{\prime} \oplus R$. This shows that $f \in L_{\varphi^{-1} \psi Q^{\prime}}\left(V^{\prime} \oplus R\right)$.

Step 4. To finish, we apply Lemma 4.6 to $\left.\varphi^{-1} \psi\right|_{Q}: Q^{\prime} \rightarrow \varphi^{-1} \psi\left(Q^{\prime}\right)$ with $V^{\prime} \oplus R$ in place of $V$ to get a product $\eta$ of $e_{i}$-reflections, taken with respect to elements of $V^{\prime} \oplus R$, such that $\left.\eta\right|_{Q^{\prime}}=\varphi^{-1} \psi$. Since $L_{Q^{\prime \prime}}\left(V^{\prime} \oplus R\right)=0$, reflections taken with respect to elements of $V^{\prime} \oplus R$ fix $Q^{\prime \prime}$. Thus, $\left.\eta\right|_{Q^{\prime \prime}}=\operatorname{id}_{Q^{\prime \prime}}$, so $\left.\eta\right|_{Q}=\varphi^{-1} \psi$. This means $\psi=\left.\varphi \eta\right|_{Q}$ and we are done.

Remark 4.7. Theorem 4.5 actually holds when (2c) is replaced with the milder conditions:

$\left(2 c^{\prime}\right)$ If $D_{i} \cong \mathbb{F}_{2} \times \mathbb{F}_{2}$ and $n_{i}=1$, then there is no $z \in V_{(i)}=V_{i}$ satisfying $h_{\beta_{i}}(z, z)=\varepsilon_{i}=1_{A_{i}}$ and $\left[\left.\beta_{i}\right|_{z^{\perp} \cap V_{i}}\right]=[0]$.

$\left(2 \mathrm{c}^{\prime \prime}\right)$ If $D_{i} \cong \mathbb{F}_{2} \times \mathbb{F}_{2}$ and $n_{i}>1$, then $Q_{i} ¥ \varepsilon_{i} A_{i}$.

The idea is to extend Lemma 4.6 to the case $D_{i} \cong \mathbb{F}_{2} \times \mathbb{F}_{2}, n_{i}>1$, and $Q \cong e_{i}^{\prime} A$, where $e_{i}^{\prime}$ is a lifting of the idempotent $\varepsilon_{i}^{\prime}=e_{11}+e_{22} \in A_{i}$. The proof requires using both $e_{i}$-reflections (to get $\bar{x}=\bar{y}$ ) and $e_{i}^{\prime}$-reflections (to find $\bar{z}$ and $\bar{c}$; use [20, Lm. 3.6]). The inductive step of Theorem 4.5 should also be modified. In case $D_{i} \cong \mathbb{F}_{2} \times \mathbb{F}_{2}$ and $n_{i}>1$, one chooses $V^{\prime}$ isomorphic to $e_{i}^{\prime} A$ rather than $e_{i} A$. If this results in $Q_{i}^{\prime \prime} \cong \varepsilon_{i} A_{i}$, then one should split $Q^{\prime} \cong e_{i}^{\prime} A$ into two copies of $e_{i} A$ and apply the induction hypothesis to the direct sum of $Q^{\prime \prime}$ and one of these copies. The full and detailed argument would overload the document while not benefiting any result beside Theorem 4.5 and Corollary 4.9 below, so we chose to omit it.

If we do not require in Theorem 4.5 that the extension of $\psi$ will be a product of quasi-reflections, then conditions (1c), (2a), (2b), (2c) can be dropped.

Theorem 4.8. Let $(P,[\beta])$ be a quadratic space, let $Q, S, V$ be summands of $P$, and let $\psi:\left(Q,\left[\left.\beta\right|_{Q}\right]\right) \rightarrow\left(S,\left[\left.\beta\right|_{S}\right]\right)$ be an isometry. Assume that

(1a) $L_{Q}(V)=Q^{*}$ and $L_{S}(V)=S^{*}$, and

(1b) $\psi x-x \in V$ for all $x \in V$.

Then $\psi$ extends to an isometry $\varphi \in O(P,[\beta])$ such that $\varphi x-x \in V$ for all $x \in P$.

Proof. The proof is essentially the same as the proof of [20, Th. 6.2]. However, since there are some differences in the conditions to be checked, we recall the argument.

Assume first that (1c) holds. Let $T$ be a free $A$-module with basis $\{z, w\}$. We choose $a \in A$ such that: 
- $a_{i}:=\pi_{i} a=0$ if $D_{i} \nsucceq \mathbb{F}_{2} \times \mathbb{F}_{2}$, or $D \cong \mathbb{F}_{2} \times \mathbb{F}_{2}$ and $L_{\left[\beta_{(i)}\right], V_{(i)}}\left(V_{(i)}\right) \supsetneqq A_{(i)}$.

- $a_{i}:=\pi_{i} a \in A_{(i)} \backslash\left\{0, \varepsilon_{i}\right\}$ if $D_{i} \cong \mathbb{F}_{2} \times \mathbb{F}_{2}$ and $L_{\left[\beta_{(i)}\right], V_{(i)}}\left(V_{(i)}\right) \cong A_{(i)}$.

We define a sesquilinear form $\gamma: T \times T \rightarrow A$ by linearly extending

$$
\begin{aligned}
\gamma(z, z) & =0 & \gamma(z, w) & =1 \\
\gamma(w, z) & =0 & \gamma(w, w) & =a
\end{aligned}
$$

Let $\left(P^{\prime},\left[\beta^{\prime}\right]\right)=(P,[\beta]) \perp(T,[\gamma]), Q^{\prime}=Q \oplus z A, S^{\prime}=S \oplus z A, V^{\prime}=V \oplus(z+w) A$ and define $\psi^{\prime}: Q^{\prime} \rightarrow S^{\prime}$ by $\psi^{\prime}(x \oplus z c)=\psi x \oplus z c(c \in A)$. It is easy to check that $\psi^{\prime}$ is an isometry from $\left[\left.\beta^{\prime}\right|_{Q^{\prime}}\right]$ to $\left[\left.\beta^{\prime}\right|_{S^{\prime}}\right]$. Furthermore, the conditions of Theorem 4.5 hold for $\left(P^{\prime},\left[\beta^{\prime}\right]\right), Q^{\prime}, S^{\prime}, V^{\prime}, \psi^{\prime}$ :

(1a) holds since $h_{\beta^{\prime}}(0 \oplus(z+w), 0 \oplus z)=u \in A^{\times}$,

(1b) is straightforward,

(1c) holds since $Q^{*} \cong Q^{*} \oplus A^{*} \cong Q \oplus A \cong Q^{\prime}$,

(2a) holds since $\hat{\gamma}_{(i)}\left(\pi_{i}\left(z e_{i}+w e_{i}\right)\right)=\varepsilon_{i}+a_{i}+\Lambda_{(i)}$,

(2b) holds since $\pi_{i}\left(0 \oplus\left(z e_{i}+w e_{i}\right)\right) \in V_{(i)}^{\prime} \cap V_{(i)}^{\prime \perp}$ when $D_{i} \cong \mathbb{F}_{2}$ (because then $\left.h_{\gamma_{i}}\left(\pi_{i}\left(z e_{i}+w e_{i}\right), \pi_{i}\left(z e_{i}+w e_{i}\right)\right)=2 \varepsilon_{i}=0\right)$, and

(2c) holds by Lemma 4.4, because if $D_{i} \cong \mathbb{F}_{2} \times \mathbb{F}_{2}$, then $L_{\left[\beta_{(i)}^{\prime}\right], V_{(i)}^{\prime}}\left(V_{(i)}^{\prime}\right) \nsucceq A_{(i)}$. Indeed, we have $L_{\left[\beta_{(i)}^{\prime}\right], V_{(i)}^{\prime}}\left(V_{(i)}^{\prime}\right) \cong L_{\left[\beta_{(i)}\right], V_{(i)}}\left(V_{(i)}\right) \oplus\left(a_{i}+a_{i}^{\sigma}\right) A$ because $h_{\gamma_{(i)}}\left(\pi_{i}\left(z e_{i}+w e_{i}\right), \pi_{i}\left(z e_{i}+w e_{i}\right)\right)=a_{i}+a_{i}^{\sigma}$.

Thus, $\psi^{\prime}$ extends to $\varphi^{\prime} \in O\left(P^{\prime},\left[\beta^{\prime}\right]\right)$ with $\varphi^{\prime} x-x \in V^{\prime}$ for all $x \in P^{\prime}$. We finish by showing that $\varphi^{\prime}(P \oplus 0)=P \oplus 0$. Indeed, for $x \oplus 0 \in P \oplus 0$, we can write $\varphi^{\prime}(x \oplus 0)=y \oplus(z+w) c$ for some $y \in P, c \in A$. This implies $0=h_{\beta^{\prime}}(0 \oplus z, x \oplus 0)=$ $h_{\beta^{\prime}}\left(\varphi^{\prime}(0 \oplus z), \varphi^{\prime}(x \oplus 0)\right)=h_{\beta^{\prime}}(0 \oplus z, y \oplus(z+w) c)=c$, so $c=0$ and $\psi^{\prime}(x \oplus 0) \in P \oplus 0$.

When (1c) does not hold, choose $U \in \mathscr{P}(A)$ such that $(Q \oplus U)^{*} \cong Q \oplus U$. Define a sesquilinear form $\gamma$ on $U \oplus U^{*}$ by $\gamma(x \oplus f, y \oplus g)=f y(x, y \in U, f, g \in$ $U^{*} ;\left(U \oplus U^{*},[\gamma]\right)$ is the hyperbolic quadratic space associated with $\left.U\right)$. Now, let $\left(P^{\prime},\left[\beta^{\prime}\right]\right)=(P,[\beta]) \oplus\left(U \oplus U^{*},[\gamma]\right), Q^{\prime}=Q \oplus U \oplus 0, S^{\prime}=S \oplus U \oplus 0, V^{\prime}=V \oplus 0 \oplus U^{*}$, and define $\psi^{\prime}: Q^{\prime} \rightarrow S^{\prime}$ by $\psi^{\prime}(x \oplus z \oplus 0)=\psi x \oplus z \oplus 0(x \in Q, z \in U)$. Conditions (1a), (1b), (1c) are easily seen to hold for $\left(P^{\prime},\left[\beta^{\prime}\right]\right), Q^{\prime}, S^{\prime}, V^{\prime}, \psi^{\prime}$, so by what we have shown above, $\psi^{\prime}$ extends to an isometry $\varphi^{\prime} \in O\left(P^{\prime},\left[\beta^{\prime}\right]\right)$ with $\varphi^{\prime} x-x \in V^{\prime}$. Similar arguing implies that $\varphi^{\prime}$ maps $P$ into itself.

The following corollaries can be viewed as analogues of Witt's Extension Theorem and Witt's Cancellation Theorem.

Corollary 4.9. Let $(P,[\beta])$ be a quadratic space and let $Q, S$ be summands of $P$. Assume that $(P,[\beta])$ or $\left(Q,\left[\left.\beta\right|_{Q}\right]\right)$ is unimodular. Then any isometry of $\psi$ : $\left(Q,\left[\left.\beta\right|_{Q}\right]\right) \rightarrow\left(S,\left[\left.\beta\right|_{S}\right]\right)$ extends to an isometry $\varphi \in O(P,[\beta])$.

Proof. Take $V=P$ in Theorem 4.8 Condition (1b) is clear, and condition (1a) follows from the unimodularity assumption.

Corollary 4.10. Let $(P,[\beta]),\left(Q_{1},\left[\gamma_{1}\right]\right),\left(Q_{2},\left[\gamma_{2}\right]\right)$ be quadratic spaces such that $(P,[\beta])$ is unimodular and $(P,[\beta]) \perp\left(Q_{1},\left[\gamma_{1}\right]\right) \cong(P,[\beta]) \perp\left(Q_{2},\left[\gamma_{2}\right]\right)$. Then $\left(Q_{1},\left[\gamma_{1}\right]\right) \cong\left(Q_{2},\left[\gamma_{2}\right]\right)$.

Proof. Identify $(Z,[\zeta]):=(P,[\beta]) \perp\left(Q_{1},\left[\gamma_{1}\right]\right)$ with $(P,[\beta]) \perp\left(Q_{2},\left[\gamma_{2}\right]\right)$. Corollary 4.9 implies that any isometry between the two copies of $(P,[\beta])$ in $(Z,[\zeta])$ extends to an isometry of $(Z,[\zeta])$. This isometry must map $\left(Q_{1},\left[\gamma_{1}\right]\right)$ isometrically onto $\left(Q_{2},\left[\gamma_{2}\right]\right)$. 
Corollary 4.11. Let $(P,[\beta])$ be a unimodular quadratic space. Assume that

(1) if $\left(A_{i}, \sigma_{i}, u_{i}, \Lambda_{i}\right)$ is split-orthogonal, then $D_{i} ¥ \mathbb{F}_{2}$, and

(2) if $D_{i} \cong \mathbb{F}_{2} \times \mathbb{F}_{2}$, then $P_{i} ¥ \varepsilon_{i} A_{i}$.

Then $O(P,[\beta])$ is generated by quasi-reflections.

Proof. It is enough to show that the conditions of Theorem 4.5 hold when we take $V, Q, S$ to be $P$. Indeed, conditions (1a) and (1b) hold as in the proof of Corollary 4.9. Condition (1c) holds because $L_{[\beta]}: P \rightarrow P^{*}$ is an isomorphism. To see (2a), observe that by Lemma 4.2(ii), $L_{P_{(i)}}: P_{(i)} \rightarrow P_{(i)}^{*}$ is an isomorphism, and hence either $P_{i}=0$ or $\left[\beta_{i}\right] \neq 0$. Condition $(2 \mathrm{~b})$ follows from assumption (1). Finally, assumption (2) and the unimodularity of $[\beta]$ imply, $L_{V_{(i)}}\left(V_{(i)}\right)=$ $L_{P_{(i)}}\left(P_{(i)}\right)=P_{(i)}^{*} \cong P_{(i)} \nsucceq A_{(i)}$, so condition (2c) holds by Lemma 4.4

Remark 4.12. Theorems 4.5 and 4.8 should be compared with Theorems 6.1 and 6.2 of [20] (and also Theorems 4.1, and 5.1 in [20]).

Theorem 6.1 in 20 is similar to Theorem 4.5 but it applies to the broader class of semilocal rings, and it derives the stronger conclusion that $\varphi$ is is a product of reflections (rather than quasi-reflections). However, it assumes the stronger assumptions

$\left(1 \mathrm{c}^{\prime}\right) Q$ and $S$ are free,

$\left(2 \mathrm{a}^{\prime}\right)$ if $\left(A_{i}, \sigma_{i}, u_{i}, \Lambda_{i}\right)$ is split-orthogonal, then $\left[\left.\beta_{i}\right|_{Q_{i}^{\perp} \cap V_{i}}\right] \neq[0]$,

in place of (1c) and (2a), and condition (2c) is replaced with condition $\left(2 \mathrm{c}^{\prime}\right)$ of Remark 4.7 (note that $\left(1 \mathrm{c}^{\prime}\right)$ and $\left(2 \mathrm{c}^{\prime}\right)$ imply $\left.\left(2 \mathrm{c}^{\prime \prime}\right)\right)$. In section 5, we shall give a precise description of which isometries are products of reflection (in case $A$ is semiperfect) from which it will follow that condition $\left(2 \mathrm{a}^{\prime}\right)$ cannot be completely removed in general.

Theorem 6.1 in 20 resembles Theorem 4.8. It applies to semilocal unitary rings, but it assumes condition $\left(2 \mathrm{a}^{\prime}\right)$ in addition to (1a) and (1b). This additional assumption does not allow one to derive Corollaries 4.9 and 4.10 from [20, Th. 6.2], and in fact, these corollaries are false for semilocal rings, as demonstrated by Keller [11, §2]. Nevertheless, [20, Th. 6.2] still implies Corollary 4.10 in case $\left[\left(\gamma_{1}\right)_{i}\right] \neq 0$ whenever $\left(A_{i}, \sigma_{i}, u_{i}, \Lambda_{i}\right)$ is split-orthogonal. See also [11, Th. 3.4] for different proof and strengthening of this statement.

Remark 4.13. Condition (2b) in Theorem 4.5 (and also [20, Th. 6.1]) cannot be completely removed, even when $(P,[\beta])$ is unimodular. Indeed, over $\mathbb{F}_{2}$, there is a unimodular quadratic form of dimension 4 whose isometry group is not generated by quasi-reflections (which are just reflections in this case). However, up to isomorphism, this is the only exception over $\mathbb{F}_{2}$ (see [24, Cr. 11.42], for instance). We do not know if condition (2c) can be removed in general.

$\S 4.4$. Applications. We now use the previous results to derive several consequences to hermitian categories and systems of sesquilinear forms. Hermitian categories are a purely categorical framework to work with quadratic forms. We refer the reader to [19, $\S 1],[23, \mathrm{Ch} .7]$ or [15, Ch. II] for the relevant definitions.

Let $(\mathscr{C}, *, \omega)$ be a hermitian category with form parameter $(\varepsilon, \Lambda)$. In [19, $\S 3.4]$, Quebbemann, Scharlau and Schulte prove that unimodular $(\varepsilon, \Lambda)$-quadratic forms over $\mathscr{C}$ cancel from orthogonal sums, provided the following assumptions hold:

(A) All idempotent morphisms in $\mathscr{C}$ split. 
(B) Every object of $\mathscr{C}$ is a direct sum of finitely many objects with local endomorphism ring (or, alternatively, the endomorphism ring of every object in $\mathscr{C}$ is semiperfect).

(C) For every $M \in \mathscr{C}, E:=\operatorname{End}_{\mathscr{C}}(M)$ is complete in the $\operatorname{Jac}(E)$-adic topology. After proving this consequence, the authors comment that condition $(\mathrm{C})$ is in fact unnecessary since one can apply transfer (see [19, Pr. 2.4]) to translate the problem to cancellation of quadratic forms over semiperfect unitary rings, and then apply Reiter's Theorem 6.2 in [20]. This also implies that condition (A) is unnecessary. However, the authors seem unaware that Reiter's Theorem does not imply cancellation in general (see Remark 4.12), and hence this argument implies cancellation only in certain cases (e.g. when all three $(\varepsilon, \Lambda)$-quadratic spaces involved are unimodular and their base objects are progenerators of $\mathscr{C})$.

Nevertheless, by replacing [20, Th. 6.2] with Theorem 4.8 (or Corollary 4.10), we see that condition $(\mathrm{C})$ can indeed be dropped. We have therefore obtained:

Corollary 4.14. Assume condition $(B)$ is satisfied. Then unimodular $(\varepsilon, \Lambda)$ quadratic forms over $\mathscr{C}$ cancel from orthogonal sums.

By the same methods (i.e. via transfer in the sense of [19, Pr. 2.4]), Corollary 4.9 implies:

Corollary 4.15. Assume condition $(B)$ is satisfied, let $(P,[\beta])$ be a unimodular $(\varepsilon, \Lambda)$-quadratic space, and let $Q, S$ be summands of $P$. Then any isometry $\psi$ : $\left(Q,\left[\left.\beta\right|_{Q}\right]\right) \rightarrow\left(S,\left[\left.\beta\right|_{S}\right]\right)$ extends to an isometry $\varphi \in O(P,[\beta])$.

We now combine Corollary 4.14 with results from [5, [7] and [6] to obtain cancellation results for systems of (not-necessarily unimodular) sesquilinear forms. Henceforth, let $R$ be a commutative ring, and let $\mathscr{C}$ be an $R$-category equipped with $R$-linear hermitian structures $\left\{*_{i}, \omega_{i}\right\}_{i \in I}$; see [6, $\left.\S 2.4, \S 4\right]$ for the definitions.

Corollary 4.16. Assume that $R$ is local and henselian, $2 \in R^{\times}$, and at least one of the following holds:

(1) $R$ is noetherian and $\operatorname{End}_{\mathscr{C}}(M)$ is finitely generated as an $R$-module for all $M \in \mathscr{C}$

(2) $R$ is a valuation ring, and $\operatorname{End}_{\mathscr{C}}(M)$ is $R$-torsion-free and has finite rank for all $M \in \mathscr{C}$.

Then systems of sesquilinear forms over $\left(\mathscr{C},\left\{*_{i}, \omega_{i}\right\}_{i \in I}\right)$ cancel from orthogonal sums.

Proof. This is similar to the proof of [6, Th. 5.2]. By [6, Th. 4.1], the category of sesquilinear forms over $\left(\mathscr{C},\left\{*_{i}, \omega_{i}\right\}_{i \in I}\right)$ is equivalent to the category of unimodular 1-hermitian forms over another hermitian category $(\mathscr{D}, *, \omega)$, where $\mathscr{D}$ is the category $A \widetilde{r}_{2 I}(\mathscr{C})$ constructed in $[\underline{6}, \S 4]$. It therefore enough to prove that unimodular 1-hermitian forms over $(\mathscr{D}, *, \omega)$ cancel from orthogonal sums. Note that since $2 \in R^{\times}$, there is only one form parameter $(1, \Lambda)$ for $(\mathscr{D}, *, \omega)$, and 1-hermitian forms and $(1, \Lambda)$-quadratic forms are essentially the same. By construction, the endomorphism ring of an object $Z \in \mathscr{D}$ is a sub- $R$-algebra of $\operatorname{End}_{\mathscr{C}}(M) \times \operatorname{End}_{\mathscr{C}}(N)^{\mathrm{op}}$ for some $M, N \in \mathscr{C}$, so by Corollary 4.14, it is enough to check that such rings are semiperfect. This is indeed the case by Proposition 2.13.

As a special case of Corollary 4.16, we get: 
Corollary 4.17. Let $A$ be an $R$-algebra and let $\left\{\sigma_{i}\right\}_{i \in I}$ be a system of $R$-involutions on A. Assume that is $R$ local and henselian, $2 \in R^{\times}$, and at least one of the following holds:

(1) $R$ is noetherian and $A$ is finitely generated as an $R$-module.

(2) $R$ is a valuation ring, and $A$ is $R$-torsion-free of finite rank.

Then systems of sesquilinear forms over $\left(A,\left\{\sigma_{i}\right\}\right)$ (that are defined on f.g. projective $A$-modules) cancel from orthogonal sums.

Finally, we use [5] to show that non-unimodular hermitian forms over nonhenselian valuation rings cancel from orthogonal sums. Here, the prefix "non-" stands for "not-necessarily" and not for absolute negation.

Corollary 4.18. Let $(R, \sigma)$ be an arbitrary valuation ring with involution, and let $u \in R$ be such that $u^{\sigma} u=1$. Suppose that there exists $r \in R$ with $r+r^{\sigma} u \in R^{\times}$ (e.g. if $\left.1+u \in R^{\times}\right)$. Then non-unimodular hermitian forms over $(R, \sigma)$ cancel from orthogonal sums.

Proof. Write $v=r+r^{\sigma} u$. Then $v \in R^{\times}$and $v^{\sigma} u=v$. By conjugating with $v^{-1}$ (see Proposition 2.4) and replacing $r$ with $r v^{-1}$, we may assume that $u=1$ and $r+r^{\sigma}=1$.

By [5, Th. 4.1], the category of arbitrary 1-hermitian forms over $(R, \sigma)$ is equivalent to the category of unimodular 1-hermitian forms over a hermitian category $(\mathscr{D}, *, \omega)$ (see [5, $\S 3]$ for its definition). The category $\mathscr{D}$ is the category of morphisms in $\mathscr{P}(R)$, denoted $\operatorname{Mor}(\mathscr{P}(R)$ ). (One can also use [6, Th. 3.2] to get essentially the same result.) Notice that the Hom-sets of $\mathscr{D}$ are $R$-modules and it not hard to check that $(f \cdot a)^{*}=f \cdot a^{\sigma}$ for every morphism $f$ in $\mathscr{D}$ and $a \in R$. Since $r+r^{\sigma}=1$, the map $(Q, h) \mapsto(Q,[r h])$ taking 1-hermitian forms over $\mathscr{D}$ to $\left(1, \Lambda^{\mathrm{min}}\right)$-quadratic spaces over $\mathscr{D}$ is an isomorphism of categories (and there is only one form parameter $(1, \Lambda)$ for $(\mathscr{D}, *, \omega))$. Now, by Corollary 4.14 , it is enough to prove that every object of $\operatorname{Mor}(\mathscr{P}(R))$ is the direct sum of objects with local endomorphism rings.

Recall that an object of $\operatorname{Mor}(\mathscr{P}(R))$ consists of a triple $\left(P, f, P^{\prime}\right)$ such that $P, P^{\prime} \in \mathscr{P}(R)$ and $f \in \operatorname{Hom}_{R}\left(P, P^{\prime}\right)$. The endomorphism ring of $\left(P, f, P^{\prime}\right)$ is the set of pairs $\left(g, g^{\prime}\right) \in \operatorname{End}_{R}(P) \times \operatorname{End}_{R}\left(P^{\prime}\right)$ such that $g^{\prime} f=f g$. Using the fact that $R$ is a valuation ring, one can show that every $\left(P, f, P^{\prime}\right)$ is the direct sum of objects of the form $(R, 0,0),(0,0, R)$ and $(R, t, R)$ with $t \neq 0$ : The proof essentially boils down to showing that for every matrix $f=\left(f_{i j}\right) \in \mathrm{M}_{n \times m}(R)$ there are invertible matrices $w \in \mathrm{M}_{n}(R)$ and $w^{\prime} \in \mathrm{M}_{m}(R)$ such that $\left(a_{i j}\right):=w f w^{\prime}$ is diagonal (meaning that $a_{i j}=0$ for $\left.i \neq j\right)$, and this is well-known. The endomorphism rings of $(R, 0,0)$, $(0,0, R)$ and $(R, t, R)$ are easily seen to be $R$, which is local, so we are done.

\section{Generation by Reflections}

Let $(P,[\beta])$ be a unimodular quadratic space defined over a semiperfect unitary ring. Denote by $O^{\prime}(P,[\beta])$ the subgroup of $O(P,[\beta])$ generated by reflections. In this section, we describe $O^{\prime}(P,[\beta])$ and show that, apart from an obvious exception, it is a subgroup of finite index in $O(P,[\beta])$, and that index can be determined in terms of $A$ and $P$.

$\S 5.1$. Dickson's Invariant. Let $(A, \sigma, u, \Lambda)$ be an orthogonal simple artinian unitary ring (see $\S 2.4$, let $K=\operatorname{Cent}(A)$, and let $(P,[\beta])$ be a unimodular quadratic space. We now recall Dickson's Invariant (also called pseudodeterminant 
or quasideterminant), which is a group homomorphism

$$
\Delta=\Delta_{[\beta]}: O(P,[\beta]) \rightarrow \mathbb{Z} / 2 \mathbb{Z} .
$$

This homomorphism will be used in describing what is $O^{\prime}(P,[\beta])$. The map $\Delta$ is related to the reduced norm map on $E:=\operatorname{End}_{A}(P)$ via

$$
\operatorname{Nrd}_{E / K}(\psi)=(-1)^{\Delta(\psi)} \quad \forall \psi \in O(P,[\beta]),
$$

so one can use $\operatorname{Nrd}_{E / K}$ to define $\Delta$ in case char $K \neq 2$.

Unfortunately, there seems to be no reference defining Dickson's invariant directly for quadratic forms over noncommutative central simple algebras (but see [24, p. 160] for the case $A=K$, and [4, [15, §IV.5] for arbitrary commutative rings). We therefore satisfy with giving an ad-hoc definition that suits our needs, but may seem unnatural. In the end, we shall briefly explain how to obtain a more intrinsic definition by using the Dickson invariant of quadratic pairs introduced in [16, $\S 12 \mathrm{C}]$.

We start by recalling Dickson's invariant in the case $A=K$ (the orthogonality then forces $\sigma=\operatorname{id}_{K}, u=1$ and $\left.\Lambda=0\right)$. In this case, $(P,[\beta])$ is just a quadratic space in the classical sense. Dickson's invariant can then be defined by

$$
\Delta(\psi)=\operatorname{dim}_{K} \operatorname{im}(1-\psi)+2 \mathbb{Z} \quad \forall \psi \in O(P,[\beta]) .
$$

The map $\Delta$ is indeed a group homomorphism ([24, Th. 11.43]), and moreover, it is a morphism of algebraic groups over $K$ ([4). See also [16, Df. 12.12] and [10, p. 129] for alternative definitions in characteristic 2. (The equivalence of the definitions, and the identity (5.1), can be verified using the references specified. In particular, one should only verify that the definitions coincide on reflections by [24, Th. 11.39].)

Assume now that $A$ is arbitrary and let $E=\operatorname{End}_{A}(P)$. Then $E$ is a central simple $K$-algebra. We define $\Delta$ by

$$
\Delta(\psi)=\frac{\operatorname{dim}_{K}(1-\psi) E}{\operatorname{deg} E}+2 \mathbb{Z} \quad \forall \phi \in O(P,[\beta])
$$

It is easy to see that this definition agrees with the definition in the case $A=K$. However, we have to check that $\Delta$ is indeed a homomorphism of groups.

Proposition 5.1. If $A$ is non-split, then $\Delta \equiv 0$. Otherwise, $\Delta$ is a surjective group homomorphism, and furthermore, it is a homomorphism of algebraic groups defined over $K$.

Proof. Assume $A$ is non-split. If $\Delta(\psi)=1+2 \mathbb{Z}$, then $\operatorname{deg} E$ must be odd (because it divides $\operatorname{dim}_{K}(1-\psi) E$ ). On the other hand, $E$ is Brauer equivalent to $A$, which has an involution of the first kind (Proposition 2.6), and hence ind $E=\operatorname{ind} A$ is a power of two ([16, Cr. 2.6]). Thus, ind $A=1$, i.e. $A$ is split. (Compare with [13, Lm. 2.6.1].)

Assume $A$ is split. Observe that the definition of $\Delta$ depends only on the isomorphism class of the endomorphism ring $E=\operatorname{End}_{A}(P)$, which does not change under conjugation and $e$-transfer (see Propositions 2.4 and 2.5). These transitions also do not affect split-orthogonality by Proposition 2.12, so we may freely apply them. Now, by conjugation, we may assume $(A, \sigma, u, \Lambda)$ is standard (Proposition 2.6), i.e. $u=1$ and $\sigma$ is the matrix transpose involution. Let $e$ be the matrix unit $e_{11}$. By applying $e$-transfer, we may assume $A=K$. But in this case it is known that $\Delta$ is a surjective group homomorphism, and also a homomorphism of algebraic groups defined over $K$. 
We now compute the Dickson invariant of reflections.

Proposition 5.2. Let $(A, \sigma, u, \Lambda)$ be an orthogonal simple artinian unitary ring, let $e \in A$ be an idempotent, and let $(P,[\beta])$ be a unimodular quadratic space over $(A, \sigma, u, \Lambda)$ with $P \neq 0$. Then for every e-reflection $s \in O(P,[\beta])$ we have

$$
\Delta(s)=\operatorname{deg} e A e+2 \mathbb{Z} .
$$

Proof. We assume $e \neq 0$; the proposition becomes trivial otherwise. Also, if $A$ is non-split, then ind $e A e=$ ind $A$ is even, so we are done by Proposition 5.1 .

Assume $A$ is split, i.e. $A=\mathrm{M}_{n}(K)$ where $K$ is a field. By conjugation, we may assume that $\sigma$ is the transpose involution and $u=1$ (Proposition 2.6. Remark 3.6(i)). Let $\left\{e_{i j}\right\}$ be the standard matrix units of $A$. Then $e$ is equivalent to $\sum_{i=1}^{r} e_{i i}$ for some $r$. By Lemma 3.4 me replace $e$ with $\sum_{i=1}^{r} e_{i i}$ to assume $e^{\sigma}=e$. Now, by applying $e$-transfer, we may assume $e=1$ (Proposition 2.5. Remark 3.6(ii)). We further tensor $(A, \sigma, u, \Lambda)$ and $(P,[\beta])$ with an algebraic closure $F$ of $K$, to assume $K$ is algebraically closed. (Namely, we replace $(A, \sigma, u, \Lambda)$ with $\left(A \otimes_{K} F, \sigma \otimes_{K} \operatorname{id}_{F}, u \otimes 1, \Lambda \otimes_{K} F\right)$, and $(P,[\beta])$ with $\left(P \otimes_{K} F,\left[\beta_{F}\right]\right)$, where $\beta_{F}$ is defined by $\beta_{F}\left(x \otimes a, x^{\prime} \otimes a^{\prime}\right)=\beta\left(x, x^{\prime}\right) \otimes a a^{\prime}$ for all $x, x^{\prime} \in P, a, a^{\prime} \in A$. The details are left to the reader.)

Let $S$ be the set of pairs $(y, \lambda) \in P \times \Lambda^{\text {min }}$ satisfying $\beta(y, y)+\lambda \in \operatorname{GL}_{n}(K)$. Then $S$ is open in the Zaritzki topology of $P \times \Lambda$ (viewed as an affine space over $K)$, and hence connected. Since $\Delta: O(P,[\beta]) \rightarrow \mathbb{Z} / 2 \mathbb{Z}$ is a morphism of algebraic groups (Proposition 5.1), the map

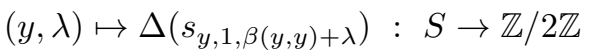

is continuous in the Zaritzki topology. Therefore, since $S$ is connected, it is enough to prove that $\Delta(s)=\operatorname{deg} A+2 \mathbb{Z}=n+2 \mathbb{Z}$ for some reflection $s$.

Let $\left\{e_{i j}\right\}_{i, j}$ be the standard matrix units of $A=\mathrm{M}_{n}(K)$. By Lemma 3.4)(i), every $e_{11}$-reflection is an $e_{i i}$-reflection for any $i$, so by Lemma 3.4(ii), the product of $n e_{11}$-reflections is a reflection. Since $\Delta$ is a group homomorphism, it is therefore enough to prove that there is an $e_{11}$-reflection $s$ with $\Delta(s)=1+2 \mathbb{Z}$. Now, note that $e_{11} A e_{11} \cong K$. Applying $e_{11}$-transfer (together with Remark 3.6(ii)), we are reduced to show that when $A=K$, there exists a reflection $s$ with $\Delta(s)=1+2 \mathbb{Z}$. But this well-established; see [16, Ex. 12.13], for instance.

Remark 5.3. Another way to define $\Delta_{[\beta]}$ when $A$ is an arbitrary central simple $K$-algebra is by relating $(P,[\beta])$ with a quadratic pair in the sense of $[16, \S 5 \mathrm{~B}]$ : By [17, $\operatorname{Pr} .4 .2] 2(P,[\beta])$ induces a quadratic pair $(\tau, f)$ on the central simple $K$-algebra $E:=\operatorname{End}_{A}(P)$, and one can show that $O(P,[\beta])$ is canonically isomorphic to

$$
O(\tau, f):=\left\{x \in E: x^{\tau} x=1 \text { and } f\left(x^{-1} a x\right)=f(a) \forall a \in E\right\} .
$$

The map $\Delta: O(P,[\beta]) \rightarrow \mathbb{Z} / 2 \mathbb{Z}$ can now be defined via the Dickson invariant of similitudes of quadratic pairs introduced in [16, $\S 12 \mathrm{C}]$. To check the equivalence of this definition with the one previously given, it is enough to show that conjugation and $e$-transfer do not affect the quadratic pair $(\tau, f)$ constructed in [17, Pr. 4.2], and that both definitions are compatible with extending to a splitting field. Provided that, it is enough to check that the definitions coincide when $A=K$, and this

\footnotetext{
${ }^{2} \operatorname{Pr} .4 .2$ of 17 is phrased in the case $A$ is a division ring, but the proof works for any central simple $K$-algebra.
} 
follows from [16, Ex. 12.31]. We leave all the details (which seem to be missing in the literature) to the reader.

§5.2. Generation by Reflections. We now use the Dickson invariant to describe the group $O^{\prime}(P,[\beta])$. Throughout, $(A, \sigma, u, \Lambda)$ is a semiperfect (and at one point semilocal) ring. We use the same setting as in $\S 4.1$ and set some further notation.

Let $(P,[\beta])$ be a quadratic space. Then any $\psi \in O(P,[\beta])$ gives rise to $\psi_{i} \in$ $O\left(P_{i},\left[\beta_{i}\right]\right)$ given by $\psi_{i}\left(\pi_{i} x\right)=\pi_{i}\left(\psi_{i} x\right)$. Observe that if $\psi$ is an $e$-reflection of $(P,[\beta])$ (with $e$ an idempotent of $A$ ), then $\psi_{i}$ is a $\pi_{i} e$-reflection of $\left(P_{i},\left[\beta_{i}\right]\right)$. In particular, if $\psi$ is a reflection, then so is $\psi_{i}$. Conversely, when the image of $e$ in $\bar{A}$ lives in $A_{i}$, every $\pi_{i} e$-reflection of $\left(P_{i},\left[\beta_{i}\right]\right)$ is induced from an $e$-reflection. This fact, which easily follows from Lemma 3.1, will be used repeatedly henceforth.

When $\left(A_{i}, \sigma_{i}, u_{i}, \Lambda_{i}\right)$ split-orthogonal and $(P,[\beta])$ is unimodular, we define

$$
\Delta_{i}=\Delta_{i,[\beta]}: O(P,[\beta]) \rightarrow \mathbb{Z} / 2 \mathbb{Z}
$$

by $\Delta_{i}(\psi)=\Delta_{\left[\beta_{i}\right]}\left(\psi_{i}\right)$ (Lemma 4.2 (ii) implies that $\left(P_{i},\left[\beta_{i}\right]\right)$ is unimodular). It is clear that $\Delta_{i}$ is a group homomorphism.

We shall call $\left(A_{i}, \sigma_{i}, u_{i}, \Lambda_{i}\right)$ odd (resp. even) split-orthogonal if $\left(A_{i}, \sigma_{i}, u_{i}, \Lambda_{i}\right)$ is split-orthogonal and $n_{i}$ is odd (resp. even; recall that $A_{i}=\mathrm{M}_{n_{i}}\left(D_{i}\right)$ ).

Finally, let $\varepsilon_{i}^{(j)}$ denote the matrix unit $e_{j j}$ in $A_{i}$. Then $\left\{\varepsilon_{i}^{(j)}\right\}_{i, j}$ is a complete system of orthogonal idempotents in $\bar{A}$, hence it can be lifted to a complete system of orthogonal idempotents $\left\{e_{i}^{(j)}\right\}_{i, j}$ in $A$. We choose this lifting to have $e_{i}=e_{i}^{(1)}$ (where $e_{i}$ is defined as in $\S 4.1$ ), and set $f_{i}=\sum_{j} e_{i}^{(j)}$ (so $\bar{f}_{i}=1_{A_{i}}$ ).

Lemma 5.4. Let $\left(P_{i},\left[\beta_{i}\right]\right)$ be a unimodular quadratic space over $\left(A_{i}, \sigma_{i}, u_{i}, \Lambda_{i}\right)$.

(i) If $\left(A_{i}, \sigma_{i}, u_{i}, \Lambda_{i}\right)$ is not split-orthogonal, then $\operatorname{id}_{P}$ is an $e_{i}$-reflection of $(P,[\beta])$.

(ii) If $P_{i} \neq 0$, then $\left(P_{i},\left[\beta_{i}\right]\right)$ admits an $\varepsilon_{i}$-reflection.

Proof. (i) If $\left(A_{i}, \sigma_{i}, u_{i}, \Lambda_{i}\right)$ is not split-orthogonal, then so is $\left(A_{(i)}, \sigma_{(i)}, u_{(i)}, \Lambda_{(i)}\right)$ (Proposition 2.12). We claim that $\Lambda_{(i)} \cap A_{(i)}^{\times} \neq \emptyset$. Indeed, if $A_{(i)} \cong E \times E^{\mathrm{op}}$ with $E$ a division ring, then $\left(1_{E}, 0_{E}^{\mathrm{op}}\right)-\left(1_{E}, 0_{E}^{\mathrm{op}}\right)_{(i)}^{\sigma} u_{(i)} \in \Lambda_{(i)} \cap A_{(i)}^{\times}$. If $A_{(i)}$ is a division ring and $\Lambda_{(i)} \cap A_{(i)}^{\times} \neq \emptyset$, then $\Lambda_{(i)}=0$. This implies, $a^{\sigma} u_{(i)}=a$ for all $a \in A_{(i)}$, and by taking $a=1$, we get $u_{(i)}=1$ and $\sigma_{(i)}=\mathrm{id}_{A^{(i)}}$. But this means $\left(A_{(i)}, \sigma_{(i)}, u_{(i)}, \Lambda_{(i)}\right)$ is split-orthogonal, a contradiction.

Now, choose $a \in e_{i}^{\sigma} \Lambda e_{i}$ such that $\pi_{i} a \in \Lambda_{(i)} \cap A_{(i)}^{\times}$. Then $a$ is $\left(e_{i}^{\sigma}, e_{i}\right)$-invertible (Lemma 3.1), hence $s_{0, e_{i}, a}$ is an $e_{i}$-reflection, and it is easy to see that $s_{0, e_{i}, a}=\mathrm{id}_{P}$.

(ii) If $\left(A_{i}, \sigma_{i}, u_{i}, \Lambda_{i}\right)$ is not split-orthogonal, take the projection to $P_{i}$ of the $e_{i}$ reflection constructed in (i). Otherwise, $A_{(i)}$ is a field and $\Lambda_{(i)}=0$, so we need to find $y \in P_{(i)}:=P_{i} \varepsilon_{i}$ with $\hat{\beta}_{(i)}(y) \neq 0$. Indeed, $[\beta]$ is unimodular, hence $\left[\beta_{i}\right]$ is unimodular (Lemma 4.2 (ii)). Since $P_{i} \neq 0$, this means $\left[\beta_{i}\right] \neq 0$, and hence $\left[\beta_{(i)}\right] \neq 0$. As $\Lambda_{(i)}=0$, there must be $y \in P_{(i)}$ with $\hat{\beta}_{(i)}(y) \neq 0$, as required.

Proposition 5.5. Let $(P,[\beta])$ be a unimodular quadratic space. Then the following conditions are equivalent:

(a) $(P,[\beta])$ admits a reflection.

(b) For all $i$, if $\left(A_{i}, \sigma_{i}, u_{i}, \Lambda_{i}\right)$ is odd split-orthogonal, then $P_{i} \neq 0$.

In this case, there exist $f_{i}$-reflections for all $i$. 
Proof. Observe that $(P,[\beta])$ has a reflection $\Longleftrightarrow(\bar{P},[\bar{\beta}])$ has a reflection $\Longleftrightarrow$ there exists $x \in \bar{P}$ such that $\hat{\bar{\beta}}(x) \cap \bar{A}^{\times} \neq \emptyset \Longleftrightarrow$ for all $i$, there exists $x \in P_{i}$ such that $\hat{\beta}_{i}(x) \cap A_{i}^{\times} \neq \emptyset \Longleftrightarrow\left(P_{i},\left[\beta_{i}\right]\right)$ admits a reflection $\Longleftrightarrow(P,[\beta])$ has an $f_{i}$-reflection for all $i$.

Assume (b) holds. By Lemma 5.4, if $\left(A_{i}, \sigma_{i}, u_{i}, \Lambda_{i}\right)$ is not split-orthogonal or $P_{i} \neq 0$, then $\left(P_{i},\left[\beta_{i}\right]\right)$ admits an $\varepsilon_{i}$-reflection $s$. By Lemma 3.4 (i), $s$ is also an $\varepsilon_{i}^{(j)}{ }_{-}$ reflection for all $j$, hence by Lemma 3.4(ii), $s^{n_{i}}$ is a reflection of $\left(P_{i},\left[\beta_{i}\right]\right)$ (because $\left.1_{A_{i}}=\sum_{j=1}^{n_{i}} \varepsilon_{i}^{(j)}\right)$. If $\left(A_{i}, \sigma_{i}, u_{i}, \Lambda_{i}\right)$ is even split-orthogonal, then $D_{i}$ is a field, $n_{i}$ is even, $\sigma_{i}$ is the matrix transpose involution, and $u=1$. It is then easy to see that $\Lambda_{i}$ contains a unit $a \in A_{i}^{\times}$, so $s_{0,1, a}$ is a reflection of $\left(P_{i},\left[\beta_{i}\right]\right)$.

Assume (b) is false. Then there is $i$ such that $\left(A_{i}, \sigma_{i}, u_{i}, \Lambda_{i}\right)$ is odd splitorthogonal and $P_{i}=0$. Thus, $\left(P_{i},\left[\beta_{i}\right]\right)$ has a reflection if and only if $\Lambda_{i}$ contains units. Since $\Lambda_{i}$ consists of $n_{i} \times n_{i}$ alternating matrices and $n_{i}$ is odd, $\Lambda_{i}$ does not contain any units and hence $\left(P_{i},\left[\beta_{i}\right]\right)$ has no reflections.

Remark 5.6. Proposition 5.5$]$ can also be proved using the more general result [20, Lm. 3.6].

Lemma 5.7. Let $(P,[\beta])$ be a unimodular quadratic space such that $P_{k} \neq 0$ whenever $\left(A_{k}, \sigma_{k}, u_{k}, \Lambda_{k}\right)$ is odd split-orthogonal. Then the product of two $e_{i}$-reflections of $(P,[\beta])$ equals a product of two reflections.

Proof. By Proposition [5.5 there exist an $f_{k}$-reflection $s_{k}$ for all $1 \leq k \leq \ell$. Let $t$ and $t^{\prime}$ be $e_{i}$-reflections. By Lemma $3.4(\mathrm{i}), t$ and $t^{-1}$ are also $\bar{e}_{i}^{(j)}$-reflections for all $1 \leq j \leq n_{i}$. Thus, by Lemma 3.4(ii), $\psi=t^{n_{i}} s_{1} \ldots \hat{s}_{i} \ldots s_{k}$ and $\varphi=$ $s_{k}^{-1} \ldots \hat{s}_{i}^{-1} \ldots s_{1}^{-1}\left(t^{-1}\right)^{n_{i}-1} t^{\prime}$ are reflections (here, ${ }^{\wedge}$ means omission). But $t t^{\prime}=\psi \varphi$, so we are done.

Recall that $O^{\prime}(P,[\beta])$ denotes the subgroup of $O(P,[\beta])$ generated by reflections.

Theorem 5.8. Let $(P,[\beta])$ be a unimodular quadratic space, and assume that

(1) if $\left(A_{i}, \sigma_{i}, u_{i}, \Lambda_{i}\right)$ is split-orthogonal, then $D_{i} \nsucceq \mathbb{F}_{2}$, and

(2) if $D_{i} \cong \mathbb{F}_{2} \times \mathbb{F}_{2}$, then $P_{i} \nsucceq \varepsilon_{i} A_{i}$.

Let $\mathcal{I}$ be the set of indices $1 \leq i \leq \ell$ such that $P_{i} \neq 0$ and $\left(A_{i}, \sigma_{i}, u_{i}, \Lambda_{i}\right)$ is split-orthogonal, and let $\xi:=\left(n_{i}+2 \mathbb{Z}\right)_{i \in \mathcal{I}} \in(\mathbb{Z} / 2 \mathbb{Z})^{\mathcal{I}}$. Denote by $\Delta_{\mathcal{I}}$ the group homomorphism

$$
\psi \mapsto\left(\Delta_{i}(\psi)\right)_{i \in \mathcal{I}}: O(P,[\beta]) \rightarrow(\mathbb{Z} / 2 \mathbb{Z})^{\mathcal{I}} .
$$

Then:

(i) $\Delta_{\mathcal{I}}$ is onto (even without assuming (1) and (2)).

(ii) If $P_{i} \neq 0$ whenever $\left(A_{i}, \sigma_{i}, u_{i}, \Lambda_{i}\right)$ is odd split-orthogonal, then $O^{\prime}(P,[\beta])=$ $\Delta_{\mathcal{I}}^{-1}(\{0, \xi\})$.

(ii) If there is $i$ such that $P_{i}=0$ and $\left(A_{i}, \sigma_{i}, u_{i}, \Lambda\right)$ is odd split-orthogonal, then $O^{\prime}(P,[\beta])=1$.

Proof. (i) Let $j \in \mathcal{I}$. By Proposition 5.2, $\Delta_{\mathcal{I}}$ of an $e_{j}$-reflection is $\left(\delta_{i j}\right)_{i \in \mathcal{I}}$ (where $\delta_{i j}=1$ if $i=j$ and 0 otherwise), and by Lemma 5.4(ii), $e_{j}$-reflections exist for all $i \in \mathcal{I}$, so $\Delta_{\mathcal{I}}$ is onto.

(ii) By Proposition [5.5. $(P,[\beta])$ admits a reflection $s$, and by Proposition 5.2, $\Delta_{\mathcal{I}}(s)=\xi$. This also implies that $O^{\prime}(P,[\beta]) \subseteq \Delta_{\mathcal{I}}^{-1}(\{0, \xi\})$. Now, to prove the other inclusion, it enough to show that $\operatorname{ker} \Delta_{\mathcal{I}} \subseteq O^{\prime}(P,[\beta])$. Let $\psi \in \operatorname{ker} \Delta_{\mathcal{I}}$. By 
URIYA A. FIRST

Corollary 4.11, $\psi$ is a product of quasi-reflections. Moreover, from the proof of Theorem 4.5 it follows that $\psi$ can written as a product of $e_{1}$-reflections followed by a product of $e_{2}$-reflection etc., and if $P_{i}=0$, then the product includes no $e_{i}$ reflections. For $1 \leq i \leq \ell$ with $P_{i} \neq 0$, let $m_{i}$ denote the number of $e_{i}$-reflection used to express $\psi$. We claim that $m_{i}$ can be taken to be even. By Lemma 5.7] this will imply $\psi \in O^{\prime}(P,[\beta])$. Indeed, if $\left(A_{i}, \sigma_{i}, u_{i}, \Lambda_{i}\right)$ is split-orthogonal (and $P_{i} \neq 0$ ), then by Proposition $5.2, m_{i}+2 \mathbb{Z}=\Delta_{i}(\psi)=0$, so $m_{i}$ is even. When $\left(A_{i}, \sigma_{i}, u_{i}, \Lambda_{i}\right)$ is not split-orthogonal, we can freely increase $m_{i}$ by inserting id ${ }_{P}$, which is an $e_{i}$-reflection by Lemma 5.4(i), into the product, so again, $m_{i}$ can be made even.

(iii) This follows from Proposition 5.5

Corollary 5.9. Let $n$ (resp. $m$ ) denote the number of $i$-s such that $\left(A_{i}, \sigma_{i}, u_{i}, \Lambda_{i}\right)$ is odd (resp. even) split-orthogonal. Then for any unimodular quadratic space $(P,[\beta])$ admitting a reflection (see Proposition 5.5), we have

$$
\left[O(P,[\beta]): O^{\prime}(P,[\beta])\right]=2^{m+\max \{n-1,0\}} .
$$

We believe that Theorem 5.8(ii) should also be true when $A$ is semilocal and $P$ is a progenerator (i.e. $A$ is a summand of $P^{n}$ for some $A$, or equivalently $P_{i} \neq 0$ for all $i$ ). Indeed, we have the following.

Theorem 5.10. Provided $P$ is free, part (ii) of Theorem5.8 holds under the milder assumption that $A$ is semilocal.

Proof. By Proposition $[5.5,(\bar{P},[\bar{\beta}])$ admits a reflection, which can lifted to a reflection $s$ of $(P,[\beta])$. That reflection satisfies $\Delta_{\mathcal{I}}(s)=\xi$ by Proposition 5.2. This proposition also implies that $O^{\prime}(P,[\beta]) \subseteq \Delta_{\mathcal{I}}^{-1}(\{0, \xi\})$, so again, it is left to prove that ker $\Delta_{\mathcal{I}} \subseteq O^{\prime}(P,[\beta])$. Let $\psi \in \operatorname{ker} \Delta_{\mathcal{I}}$, and let $\bar{\psi}$ be the isometry it induces on $(\bar{P},[\bar{\beta}])$ (namely, $\bar{\psi}(\bar{x})=\overline{\psi x}$ ). Arguing as in the proof of Theorem 5.8 , we see that

$\bar{\psi}$ is a product of reflections of $(\bar{P},[\bar{\beta}])$. These reflections can be lifted to reflections of $(P,[\beta])$, and their product is an isometry $\psi^{\prime} \in O(P,[\beta])$ such that $\bar{\psi}=\overline{\psi^{\prime}}$. Replacing $\psi$ with $\psi^{\prime-1} \psi$, we may assume $\bar{\psi}=\mathrm{id}_{\bar{P}}$, or rather, $\psi x-x \in P \operatorname{Jac}(A)$ for all $x \in P$. Now, it is shown in the proof of [20, Th. 6.2] that such $\psi$ is a product of reflections (here we need $P$ to be free), so we are done. (Notice that [20. Th. 6.2] assumes that $\left[\beta_{i}\right] \neq 0$ whenever $\left(A_{i}, \sigma_{i}, u_{i}, \Lambda_{i}\right)$ is split-orthogonal, but the argument that we have counted on only needs $\left[\beta_{i}\right] \neq 0$ when $\left(A_{i}, \sigma_{i}, u_{i}, \Lambda_{i}\right)$ is odd split-orthogonal. Also note that $\left[\beta_{i}\right] \neq 0 \Longleftrightarrow P_{i} \neq 0$ because $\left(P_{i},\left[\beta_{i}\right]\right)$ is unimodular.)

Example 5.11. Part (i) of Theorem 5.8 may fail when $A$ is only assumed to be semilocal. For example, take $A$ to be a non-local semilocal commutative domain with $2 \in A^{\times}$, set $\sigma=\operatorname{id}_{A}, u=1, \Lambda=0$, and define $\beta: A \times A \rightarrow A$ by $\beta(x, y)=x y$. It is easy to check that $O(A,[\beta])=\left\{ \pm \mathrm{id}_{A}\right\}$. However, $|\mathcal{I}|>1$ because $\bar{A}$ is not a field, and hence $\Delta_{\mathcal{I}}$ cannot be onto.

\section{REFERENCES}

[1] Gorô Azumaya. On maximally central algebras. Nagoya Math. J., 2:119-150, 1951.

[2] Anthony Bak. On modules with quadratic forms. In Algebraic K-Theory and its Geometric Applications (Conf., Hull, 1969), pages 55-66. Springer, Berlin, 1969. 
[3] Hyman Bass. Unitary algebraic K-theory. In Algebraic K-theory, III: Hermitian K-theory and geometric applications (Proc. Conf., Battelle Memorial Inst., Seattle, Wash., 1972), pages 57-265. Lecture Notes in Math., Vol. 343. Springer, Berlin, 1973.

[4] Hyman Bass. Clifford algebras and spinor norms over a commutative ring. Amer. J. Math., 96:156-206, 1974.

[5] Eva Bayer-Fluckiger and Laura Fainsilber. Non-unimodular Hermitian forms. Invent. Math., 123(2):233-240, 1996.

[6] Eva Bayer-Fluckiger, Uriya A. First, and Daniel A. Moldovan. Hermitian categories, extension of scalars and systems of sesquilinear forms. Pacific J. Math., 270(1):1-26, 2014.

[7] Eva Bayer-Fluckiger and Daniel Arnold Moldovan. Sesquilinear forms over rings with involution. J. Pure Appl. Algebra, 218(3):417-423, 2014.

[8] Uriya A. First. General bilinear forms. Israel J. Math., 205(1):145-183, 2015.

[9] Uriya A. First. Rings that are Morita equivalent to their opposites. J. Algebra, 430:26-61, 2015.

[10] Larry C. Grove. Classical groups and geometric algebra, volume 39 of Graduate Studies in Mathematics. American Mathematical Society, Providence, RI, 2002.

[11] Bernhard Keller. A remark on quadratic spaces over noncommutative semilocal rings. Math. Z., 198(1):63-71, 1988.

[12] Manfred Knebusch. Isometrien über semilokalen Ringen. Math. Z., 108:255-268, 1969.

[13] M. Kneser. Lectures on Galois cohomology of classical groups. Tata Institute of Fundamental Research, Bombay, 1969. With an appendix by T. A. Springer, Notes by P. Jothilingam, Tata Institute of Fundamental Research Lectures on Mathematics, No. 47.

[14] Martin Kneser. Witts Satz über quadratische Formen und die Erzeugung orthogonaler Gruppen durch Spiegelungen. Math.-Phys. Semesterber., 17:33-45, 1970.

[15] Max-Albert Knus. Quadratic and Hermitian forms over rings, volume 294 of Grundlehren der Mathematischen Wissenschaften [Fundamental Principles of Mathematical Sciences]. Springer-Verlag, Berlin, 1991. With a foreword by I. Bertuccioni.

[16] Max-Albert Knus, Alexander Merkurjev, Markus Rost, and Jean-Pierre Tignol. The book of involutions, volume 44 of American Mathematical Society Colloquium Publications. American Mathematical Society, Providence, RI, 1998. With a preface in French by J. Tits.

[17] Max-Albert Knus and Oliver Villa. Quadratic quaternion forms, involutions and triality. In Proceedings of the Conference on Quadratic Forms and Related Topics (Baton Rouge, LA, 2001), number Extra Vol., pages 201-218 (electronic), 2001.

[18] T. Y. Lam. Lectures on modules and rings, volume 189 of Graduate Texts in Mathematics. Springer-Verlag, New York, 1999.

[19] H.-G. Quebbemann, W. Scharlau, and M. Schulte. Quadratic and Hermitian forms in additive and abelian categories. J. Algebra, 59(2):264-289, 1979.

[20] H. Reiter. Witt's theorem for noncommutative semilocal rings. J. Algebra, 35:483-499, 1975.

[21] Louis H. Rowen. Ring theory. Vol. I, volume 127 of Pure and Applied Mathematics. Academic Press Inc., Boston, MA, 1988.

[22] Amit Roy. Cancellation of quadratic form over commutative rings. J. Algebra, 10:286-298, 1968.

[23] Winfried Scharlau. Quadratic and Hermitian forms, volume 270 of Grundlehren der Mathematischen Wissenschaften [Fundamental Principles of Mathematical Sciences]. SpringerVerlag, Berlin, 1985.

[24] Donald E. Taylor. The geometry of the classical groups, volume 9 of Sigma Series in Pure Mathematics. Heldermann Verlag, Berlin, 1992.

[25] Peter Vámos. Decomposition problems for modules over valuation domains. J. London Math. Soc. (2), 41(1):10-26, 1990.

[26] C. T. C. Wall. On the axiomatic foundations of the theory of Hermitian forms. Proc. Cambridge Philos. Soc., 67:243-250, 1970.

[27] R. B. Warfield, Jr. Cancellation of modules and groups and stable range of endomorphism rings. Pacific J. Math., 91(2):457-485, 1980

Hebrew University of Jerusalem, Israel. 